\title{
ON $l$-ADIC ITERATED INTEGRALS, III GALOIS ACTIONS ON FUNDAMENTAL GROUPS
}

\author{
ZDZISŁAW WOJTKOWIAK
}

\begin{abstract}
We continue to study $l$-adic iterated integrals introduced in the first part. We shall calculate explicitly $l$-adic logarithm and $l$-adic polylogarithms. Next we shall use these results to study Galois representations on the fundamental group of $\mathbf{P} \frac{1}{\mathbf{Q}\left(\mu_{n}\right)} \backslash\left\{0, \mu_{n}, \infty\right\}$.
\end{abstract}

\section{§13. Introduction to Part III}

13.0. In Part I and II of our paper on $l$-adic iterated integrals we were studying coefficients of the power series $\Lambda_{p}(\sigma)$. We were trying to go as far as possible without explicit calculations of these coefficients. (Only in Section 7 we use explicitly the fact that the coefficients at degree one of the power series $\Lambda_{p}$ are Kummer characters, because it is well known that the realization map from $K^{*} \otimes \mathbf{Q}$ to $H^{1}\left(G_{K} ; \mathbf{Q}(1)\right)$ associates to $z \in K$ the Kummer character corresponding to $z$. We could however use Propositions 11.0.17 and 11.1.0 to avoid explicit calculations in Proposition 7.1.0.)

We start this paper with an explicit calculations of $l$-adic logarithm. We show that $l(z)$ is a Kummer character $\kappa(z)$ associated to $z$. Next we shall calculate explicitly coefficients in degree 1 of the power series $\log \Lambda_{p}$. These coefficients are also expressed by Kummer characters. We have the following result.

Theorem F. Let $X=\mathbf{P}_{K}^{1} \backslash\left\{a_{1}, \ldots, a_{n}, \infty\right\}$, let $z, v \in \hat{X}(K)$ and let $p$ be a path from $v$ to $z$. Let $g_{i}: X \rightarrow \mathbf{P}_{K}^{1} \backslash\{0, \infty\}$ be given by $g_{i}(z)=z-a_{i}$ for $i=1, \ldots, n$. Let us fix a path $q_{i}$ from $\overrightarrow{01}$ to $g_{i}(v)$ for $i=1, \ldots, n$. (If $v=\overrightarrow{a_{i} a}$ then $g_{i}(v)=\left(a-a_{i}\right) \overrightarrow{01}$ and $g_{j}(v)=a_{i}-a_{j}$ for $i \neq j$.) If

$$
\mathfrak{f}_{p} \equiv x_{1}^{\alpha_{1}} \cdots x_{n}^{\alpha_{n}} \bmod \Gamma^{2} \pi_{1}\left(X_{\bar{K}} ; v\right)
$$

then

Received January 7, 2003.

Revised December 9, 2003.

2000 Mathematics Subject Classification: 11G55, 11G99, $14 \mathrm{G} 32$. 
i) if $z, v \in X(K)$ then $\alpha_{i}=\kappa\left(z-a_{i}\right)_{g_{i}(p) \cdot q_{i}}-\kappa\left(v-a_{i}\right)_{q_{i}}$;

ii) if $z \in X(K)$ and $v=\overrightarrow{a_{i} a}$ then $\alpha_{j}=\kappa\left(z-a_{j}\right)_{g_{j}(p) \cdot q_{j}}-\kappa\left(a_{i}-a_{j}\right)_{q_{j}}$ for $i \neq j$ and $\alpha_{i}=\kappa\left(z-a_{i}\right)_{g_{i}(p) \cdot q_{i}}-\kappa\left(a-a_{i}\right)_{q_{i}}$.

We give also an explicit formula for $l$-adic polylogarithms following $[\mathrm{NW}]$. We modify slightly the proof given in $[\mathrm{NW}]$ as we shall not use a free differential calculus.

Next we are discussing $l$-adic polylogarithms evaluated at roots of unity. Let $n$ be a positive integer prime to $l$. We take $K=\mathbf{Q}\left(\mu_{n}\right)$ as our basic field. The $l$-adic polylogarithms evaluated at $n$-th roots of 1 are cocycles if paths from $\overrightarrow{01}$ to $\xi_{n}^{i}$ are chosen suitable. Next we recall some results from [W2], where we relate the $l$-adic polylogarithms evaluated at roots of unity to Soulé classes.

We show that the $l$-adic polylogarithms evaluated at $n$-th roots of unity coincide with linear combinations of Soulé classes. We also express Soulé classes by $l$-adic polylogarithms evaluated at $n$-th roots of 1 .

Using functional equations of $l$-adic polylogarithms we show that any $l_{m+1}\left(\xi_{n}^{i}\right)$ is a linear combination of $l_{m+1}\left(\xi_{n}^{k}\right)$ with $0<k<\frac{n}{2}$ and $(k, n)=1$. We conjecture that the cohomology classes $l_{m+1}\left(\xi_{n}^{k}\right)$ for $0<k<\frac{n}{2}$ and $(k, n)=1$ are linearly independent over $\mathbf{Q}_{l}$ in $H^{1}\left(G_{K} ; \mathbf{Q}_{l}(m+1)\right)$. This conjecture is equivalent to the following one.

Conjecture G. The cyclotomic elements of Soulé generate $K_{2 m-1}$ $\left(\mathbf{Z}\left[\frac{1}{l}\right]\left(\mu_{n}\right)\right) \otimes \mathbf{Q}_{l}$.

In fact we think that this is a theorem. However in the literature we found only the result concerning $K_{3}$ (see [S3, p. 246]). (There is however a proof using motives in $[\mathrm{HW}]$.)

In Section 15 we study the Galois representation

$$
G_{\mathbf{Q}\left(\mu_{n}\right)} \longrightarrow \operatorname{Aut} \pi_{1}\left(\mathbf{P} \frac{1}{\mathbf{Q}\left(\mu_{n}\right)} \backslash\left\{0, \mu_{n}, \infty\right\} ; \overrightarrow{01}\right) .
$$

Let us set $V:=\mathbf{P}_{\mathbf{Q}\left(\mu_{n}\right)}^{1} \backslash\left\{0, \mu_{n}, \infty\right\}$. Let $\mathbf{V}=\left\{X, Y_{0}, \ldots, Y_{n-1}\right\}$. As in the previous sections we embed $\pi_{1}\left(V_{\overline{\mathbf{Q}}} ; \overrightarrow{01}\right)$ into a $\mathbf{Q}_{l}$-algebra $\mathbf{Q}_{l}\{\{\mathbf{V}\}\}$ mapping $x$ (loop around 0 ) onto $e^{X}$ and each $y_{i}$ (loop around $\xi_{n}^{i}$ ) onto $e^{Y_{i}}$. Hence we get a Galois representation

$$
\varphi: G_{\mathbf{Q}\left(\mu_{n}\right)} \longrightarrow \operatorname{Aut}\left(\mathbf{Q}_{l}\{\{\mathbf{V}\}\}\right) .
$$


We recall from Part I, Section 3 that

$$
G_{m}(V, \overrightarrow{01}):=\operatorname{ker}\left(G_{\mathbf{Q}\left(\mu_{n}\right)} \rightarrow \operatorname{Aut}\left(\pi_{1}\left(V_{\overline{\mathbf{Q}}} ; \overrightarrow{01}\right) / \Gamma^{m+1} \pi_{1}\left(V_{\overline{\mathbf{Q}}} ; \overrightarrow{01}\right)\right)\right)
$$

and $G_{\infty}(V, \overrightarrow{01}):=\bigcap_{i=1}^{\infty} G_{i}(V, \overrightarrow{01})$.

The filtration $\left\{G_{m}(V, \overrightarrow{01})\right\}_{m \in \mathbf{N}}$ of $G_{1}(V, \overrightarrow{01})$ induces a filtration $\{$ Lie $\left.\left(G_{m}(V, \overrightarrow{01}) / G_{\infty}(V, \overrightarrow{01})\right)\right\}_{m \in \mathbf{N}}$ of the Lie algebra $\operatorname{Lie}\left(G_{1}(V, \overrightarrow{01}) / G_{\infty}(V, \overrightarrow{01})\right)$. Passing with the homomorphism $\varphi$ to Lie algebras and then to associated graded Lie algebras we get a morphism of associated graded Lie algebras

$$
g r(\operatorname{Lie} \varphi): \operatorname{gr}\left(\operatorname{Lie}\left(G_{1}(V, \overrightarrow{01}) / G_{\infty}(V, \overrightarrow{01})\right)\right) \otimes \mathbf{Q} \longrightarrow \operatorname{Der}^{*} \operatorname{Lie}(\mathbf{V})
$$

where $\operatorname{Lie}(\mathbf{V})$ is a free Lie algebra over $\mathbf{Q}_{l}$ on the set $\mathbf{V}$,

$$
\begin{aligned}
g r & \left(\operatorname{Lie}\left(G_{1}(V, \overrightarrow{01}) / G_{\infty}(V, \overrightarrow{01})\right)\right) \otimes \mathbf{Q} \\
& :=\bigoplus_{i=1}^{\infty}\left(\operatorname{Lie}\left(G_{i}(V, \overrightarrow{01}) / G_{i+1}(V, \overrightarrow{01})\right)\right) \otimes \mathbf{Q} \\
& \simeq \bigoplus_{i=1}^{\infty}\left(G_{i}(V, \overrightarrow{01}) / G_{i+1}(V, \overrightarrow{01})\right) \otimes \mathbf{Q}
\end{aligned}
$$

and

$$
\begin{aligned}
\operatorname{Der}^{*} \operatorname{Lie}(\mathbf{V})=\{ & D \in \operatorname{Der} \operatorname{Lie}(\mathbf{V}) \mid \\
& \forall k \in\{0, \ldots, n-1\} \exists \beta_{k}\left(X, Y_{0}, \ldots, Y_{n-1}\right) \in \operatorname{Lie}(\mathbf{V}), \\
& \left.D(X)=0 \text { and } D\left(Y_{k}\right)=\left[Y_{k}, \beta_{k}\left(X, Y_{0}, \ldots, Y_{n-1}\right)\right]\right\}
\end{aligned}
$$

(see Part I, Section 5).

TheOREM H. The image of the morphism of associated graded Lie algebras $\operatorname{gr}(\operatorname{Lie} \varphi)$ is contained in the Lie algebra of derivations

$$
\begin{aligned}
& \operatorname{Der}_{\mathbf{Z} / n}^{*} \operatorname{Lie}(\mathbf{V}):=\left\{D \in \operatorname{Der}^{*} \operatorname{Lie}(\mathbf{V}) \mid\right. \\
& \quad \exists \beta\left(X, Y_{0}, \ldots, Y_{n-1}\right) \in \operatorname{Lie}(\mathbf{V}) \forall k \in\{0, \ldots, n-1\}, \\
& \left.D(X)=0 \text { and } D\left(Y_{k}\right)=\left[Y_{k}, \beta\left(X, Y_{k}, \ldots, Y_{n-1}, Y_{0}, \ldots, Y_{k-1}\right)\right]\right\} .
\end{aligned}
$$

In the next theorem $l$-adic polylogarithms will appear. We recall that $L(\mathbf{V}):=\lim _{i}\left(\operatorname{Lie}(\mathbf{V}) / \Gamma^{i} \operatorname{Lie}(\mathbf{V})\right)$ is a free completed Lie algebra on the set $\mathrm{V}$.

We introduce the following notation. If $A$ and $B$ belong to a Lie algebra then we define $\left[[A, B] B^{0}\right]:=[A, B],\left[[A, B] B^{1}\right]:=[[A, B], B]$ and $\left[[A, B] B^{m}\right]:=\left[\left[[A, B] B^{m-1}\right], B\right]$ for $m>1$. 
TheOREM J. Let $\sigma \in G_{m}(V, \overrightarrow{01})$. If $m>1$ then $(\log \sigma)\left(Y_{0}\right)=\left[Y_{0}, \sum_{k=0}^{n-1} l_{m}\left(\xi_{n}^{n-k}\right)(\sigma)\left[\left[Y_{k}, X\right] X^{m-2}\right]\right] \bmod I_{3}+\Gamma^{m+1} L(\mathbf{V})$ where $I_{3}$ is a closed Lie ideal of $L(\mathbf{V})$ generated topologically by all Lie brackets which contain at least three $Y$ 's. If $m=1$ then

$$
(\log \sigma)\left(Y_{0}\right)=\left[Y_{0}, \sum_{k=1}^{n-1} l\left(1-\xi_{n}^{n-k}\right)(\sigma) Y_{k}\right] \bmod \Gamma^{2} L(\mathbf{V})
$$

Now we shall use functional equations of $l$-adic polylogarithms and the assumption that for each $m>0$ the $l$-adic polylogarithms $l_{m+1}\left(\xi_{n}^{i}\right)$ for $0<$ $i<\frac{n}{2}$ and $(i, n)=1$ are linearly independent over $\mathbf{Q}_{l}$ in $H^{1}\left(G_{K} ; \mathbf{Q}_{l}(m+1)\right)$. We assume that $n$ is a prime number and for traditional reason we denote it by $p$. We get the following results.

THEOREM K. Let $p$ be a prime number greater than 2 and different from $l$. Assume that for each $m>1$ the $l$-adic polylogarithms $l_{m}\left(\xi_{p}^{k}\right)$ for $0<$ $k<\frac{p}{2}$ are linearly independent over $\mathbf{Q}_{l}$ in $H^{1}\left(G_{K} ; \mathbf{Q}_{l}(m)\right)$. Then there are elements $\sigma_{1}^{k}, \sigma_{2}^{k}, \ldots, \sigma_{n}^{k}, \ldots\left(k=1, \ldots, \frac{p-1}{2}\right)$ in $\operatorname{gr} \operatorname{Lie}\left(G_{1}(V, \overrightarrow{01}) / G_{\infty}(V, \overrightarrow{01})\right)$ $\otimes \mathbf{Q}$ such that any $\sigma_{i}^{k}$ is homogenous of degree $i$ and

$$
\begin{gathered}
g r(\operatorname{Lie} \varphi)\left(\sigma_{1}^{k}\right)\left(Y_{0}\right)=\left[Y_{0}, Y_{k}+Y_{p-k}\right], \\
g r(\operatorname{Lie} \varphi)\left(\sigma_{2 i}^{k}\right)\left(Y_{0}\right) \equiv\left[Y_{0},\left[\left[Y_{k}, X\right] X^{2 i-2}\right]-\left[\left[Y_{p-k}, X\right] X^{2 i-2}\right]\right] \bmod I_{3}
\end{gathered}
$$

and

$$
\begin{aligned}
& \operatorname{gr}(\operatorname{Lie} \varphi)\left(\sigma_{2 i+1}^{k}\right)\left(Y_{0}\right) \equiv\left[Y_{0},\left(1-p^{2 i}\right)\left[\left[Y_{k}, X\right] X^{2 i-1}\right]\right. \\
& \left.+\left(1-p^{2 i}\right)\left[\left[Y_{p-k}, X\right] X^{2 i-1}\right]+2 p^{2 i}\left[\left[Y_{0}, X\right] X^{2 i-1}\right]\right] \bmod I_{3}
\end{aligned}
$$

Using Theorem $\mathrm{K}$ we show the following result.

THEOREM L. Let $p$ be a prime number greater than 3 and different from $l$. Assume that for each $m>1$ the l-adic polylogarithms $l_{m}\left(\xi_{p}^{k}\right)$ for $0<k<\frac{p}{2}$ are linearly independent over $\mathbf{Q}_{l}$ in $H^{1}\left(G_{K} ; \mathbf{Q}_{l}(m)\right)$. Let $\mathcal{S}$ be a subset of $\{1, \ldots, p-1\}$ satisfying the following conditions

i) if $k \in \mathcal{S}$ then $p-k \in \mathcal{S}$, 
ii) $(\mathcal{S}+\mathcal{S}) \cap \mathcal{S}=\emptyset$ (the sum of two elements of $\mathcal{S}$ is calculated mod $p)$.

Then the derivations $\operatorname{gr}(\operatorname{Lie} \varphi)\left(\sigma_{n}^{k_{1}}\right), \ldots, \operatorname{gr}(\operatorname{Lie} \varphi)\left(\sigma_{n}^{k_{q}}\right)$ for $\left\{k_{1}, \ldots, k_{q}\right\} \subset$ $\mathcal{S} \cap\left\{1, \ldots, \frac{p-1}{2}\right\}$ and $n=1,2, \ldots$ generate a free Lie subalgebra of the image of the Lie algebra homomorphism

$$
g r(\operatorname{Lie} \varphi): \operatorname{gr} \operatorname{Lie}\left(G_{1}(V, \overrightarrow{01}) / G_{\infty}(V, \overrightarrow{01})\right) \otimes \mathbf{Q} \longrightarrow \operatorname{Der}_{\mathbf{Z} / p}^{*} \operatorname{Lie}(\mathbf{V})
$$

and moreover these derivations are free generators of this Lie subalgebra.

\section{§14. Kummer characters}

14.0. In this subsection we shall discuss some elementary properties of Kummer characters. We shall show that an $l$-adic logarithm $l(z)$ is a Kummer character corresponding to $z$. We also calculate coefficients in degree 1 of the power series $\Lambda_{p}$. These coefficients are expressed by Kummer characters.

Let $K$ be a number field. First we state some well known and elementary results about Kummer characters without proofs.

Lemma 14.0.1. There is a bijection between compatible systems $\left\{\xi_{l^{n}}\right\}_{n \in \mathbf{N}}$ of primitive $l^{n}-t h$ roots of 1 and generators of $\pi_{1}\left(\operatorname{Spec} \bar{K}[[z]]\left[\frac{1}{z}\right] ; \overrightarrow{01}\right)$.

Let us fix a compatible system $\left\{\xi_{l^{n}}\right\}_{n \in \mathbf{N}}$ of primitive $l^{n}$-th roots of 1 . It corresponds to a generator $x$ of $\pi_{1}\left(\operatorname{Spec} \bar{K}[[z]]\left[\frac{1}{z}\right] ; \overrightarrow{01}\right)$. Let $\zeta \in K$ and let $\left\{\zeta_{n}\right\}_{n \in \mathbf{N}}$ be a compatible system of $l^{n}$-th roots of $\zeta$. We define a function

$$
\kappa \kappa_{n}(\zeta): \operatorname{Gal}(\bar{K} / K) \longrightarrow \mu_{l^{n}}
$$

in the following way. Let $\sigma \in \operatorname{Gal}(\bar{K} / K)$. We set

$$
\kappa \kappa_{n}(\zeta)(\sigma):=\sigma\left(\zeta_{n}\right) / \zeta_{n}
$$

The family $\left\{\kappa \kappa_{n}(\zeta)(\sigma)\right\}_{n \in \mathbf{N}}$ is a compatible system of $l^{n}$-th roots of 1 . Hence it follows from Lemma 14.0.1 that we get a function

$$
\kappa \kappa(\zeta): \operatorname{Gal}(\bar{K} / K) \longrightarrow \pi_{1}\left(\operatorname{Spec} \bar{K}[[z]]\left[\frac{1}{z}\right] ; \overrightarrow{01}\right)
$$

We have

$$
\kappa \kappa(\zeta)(\sigma)=x^{\kappa(\zeta)(\sigma)}
$$


for some element $\kappa(\zeta)(\sigma) \in \mathbf{Z}_{l}$. The function

$$
\kappa(\zeta): \operatorname{Gal}(\bar{K} / K) \longrightarrow \mathbf{Z}_{l}
$$

we call a Kummer character associated to $\zeta$ (and corresponding to a compatible system $\left\{\zeta_{n}\right\}_{n \in \mathbf{N}}$ of $l^{n}$-th roots of $\zeta$ ). The function $\kappa(\zeta)$ depends on a choice of a compatible system of $l^{n}$-th roots of $\zeta$.

Lemma 14.0.2. Let $\tau, \sigma \in G_{K}$. The function $\kappa(\zeta)$ satisfies

$$
\kappa(\zeta)(\tau \sigma)=\kappa(\zeta)(\tau)+\chi(\tau) \kappa(\zeta)(\sigma)
$$

Hence the function $\kappa(\zeta)$ defines a cohomology class $\kappa(\zeta) \in H^{1}\left(G_{K} ; \mathbf{Z}_{l}(1)\right)$.

LEMMA 14.0.3. Let $\left\{y_{n}\right\}_{n \in \mathbf{N}}$ be another compatible system of $l^{n}$-th roots of $\zeta$. Let $\kappa^{\prime}$ be the corresponding Kummer character. Then we have $\kappa^{\prime}=\kappa(\zeta)+a(\chi-1)$ for some $a \in \mathbf{Z}_{l}$. Moreover for any $a \in \mathbf{Z}_{l}$ there is a compatible system of $l^{n}$-th roots of $\zeta$ such that the corresponding Kummer character is equal $\kappa(\zeta)+a(\chi-1)$.

Definition 14.0.4. Any function from $G_{K}$ to $\mathbf{Q}_{l}$ of the form $\kappa(\zeta)+$ $a(\chi-1)$, where $a \in \mathbf{Q}_{l}$ we shall call also a Kummer character.

Corollary 14.0.5. i) The function

$$
\kappa(\zeta)_{\mid \operatorname{Gal}\left(\bar{K} / K\left(\mu_{l} \infty\right)\right)}: \operatorname{Gal}\left(\bar{K} / K\left(\mu_{l \infty}\right)\right) \longrightarrow \mathbf{Z}_{l}
$$

does not depend on a choice of a compatible family of $l^{n}$-th roots of $\zeta$.

ii) For any $\tau \in G_{K}$ and $\sigma \in \operatorname{Gal}\left(\bar{K} / K\left(\mu_{l \infty}\right)\right)$ we have

$$
\kappa(\zeta)\left(\tau \cdot \sigma \cdot \tau^{-1}\right)=\chi(\tau) \kappa(\sigma)
$$

iii) Let $\alpha, \beta \in K$. Then after the restriction to $\operatorname{Gal}\left(\bar{K} / K\left(\mu_{l^{\infty}}\right)\right)$ we have

$$
\kappa(\zeta)(\alpha \cdot \beta)=\kappa(\zeta)(\alpha)+\kappa(\zeta)(\beta)
$$

We denote by $\mu(K)$ a subgroup of roots of unity in $K^{*}$.

Proposition 14.0.6. We have:

i) If $\kappa(\zeta)=0$ on $\operatorname{Gal}\left(\bar{K} / K\left(\mu_{l^{\infty}}\right)\right)$ then $\zeta$ is a root of unity. 
ii) The homomorphism $\varphi: K^{*} / \mu(K) \rightarrow \operatorname{Hom}\left(\operatorname{Gal}\left(\bar{K} / K\left(\mu_{l^{\infty}}\right)\right)\right.$; $\left.\mathbf{Q}_{l}\right) d e$ fined by $\varphi(\zeta):=\kappa(\zeta)$ is injective and it extends to an injective homomorphism $\varphi: K^{*} / \mu(K) \otimes \mathbf{Z}_{l} \rightarrow \operatorname{Hom}\left(\operatorname{Gal}\left(\bar{K} / K\left(\mu_{l^{\infty}}\right)\right) ; \mathbf{Q}_{l}\right)$.

We finish with a result that for a given finite number of $z_{1}, \ldots, z_{n} \in K^{*}$ their Kummer characters from $G_{K}$ to $\mathbf{Q}_{l}$ can be chosen in a compatible way.

Proposition 14.0.7. Let $z_{1}, \ldots, z_{n} \in K^{*}$. Then we can chose Kummer characters $\kappa\left(z_{1}\right), \ldots, \kappa\left(z_{n}\right)$ such that any relation $\sum_{i=1}^{n} z_{i} \otimes q_{i}=0$ in $K^{*} \otimes \mathbf{Q}$ implies $\sum_{i=1}^{n} q_{i} \kappa\left(z_{i}\right)=0$ in the group of one-cocycles $Z\left(G_{K} ; \mathbf{Q}_{l}(1)\right)$.

Proof. We consider a vector subspace of $K^{*} \otimes \mathbf{Q}$ generated by $z_{1} \otimes 1, \ldots, z_{n} \otimes 1$. Without lost of generality we can assume that $z_{1} \otimes$ $1, \ldots, z_{p} \otimes 1$ are linearly independent. If $z_{r} \otimes 1=\sum_{i=1}^{p} q_{i}\left(z_{i} \otimes 1\right)$ then $N\left(z_{r} \otimes 1\right)=\sum_{i=1}^{p} Q_{i}\left(z_{i} \otimes 1\right)$, where $N$ and $Q_{i}$ are integers. Observe that $N \kappa\left(z_{r}\right)=\sum_{i=1}^{p} Q_{i} \kappa\left(z_{i}\right)$ on $\operatorname{Gal}\left(\bar{K} / K\left(\mu_{l}\right)\right)$. Proposition 14.0.6 implies that $\left(\prod_{i=1}^{p} z_{i}^{Q_{i}}\right) \cdot z_{r}^{-N}$ is a root of unity. Hence $\sum_{i=1}^{p} Q_{i} \kappa\left(z_{i}\right)=$ $N \kappa\left(z_{r}\right)+k(\chi-1)$ for some $k \in \mathbf{Z}_{l}$. We replace $\kappa\left(z_{r}\right)$ by $\kappa\left(z_{r}\right)+\frac{k}{N}(\chi-1)$.

Remark 14.0.8. If $K=\mathbf{Q}$ then we can choose Kummer characters satisfying

$$
\kappa\left(z_{1} \cdot z_{2}\right)=\kappa\left(z_{1}\right)+\kappa\left(z_{2}\right)
$$

for any $z_{1}, z_{2} \in \mathbf{Q}^{*}$ in the following way. Let $z \in \mathbf{Q} \backslash\{0\}$. Let $|z|^{1 / l^{n}}$ be the positive real $l^{n}$-th root of the absolute value of $z$. Let $\xi_{l^{n}}=\exp \left(\frac{2 \pi i}{l^{n}}\right)$ and let $\sigma \in G_{\mathbf{Q}}$. We set

$$
\frac{\sigma\left(|z|^{1 / l^{n}}\right)}{|z|^{1 / l^{n}}}=\left(\xi_{l^{n}}\right)^{\kappa(z)(\sigma)} .
$$

Observe that for any $x, y \in \mathbf{Q} \backslash\{0\}$ we have

$$
\kappa(x)+\kappa(y)=\kappa(x \cdot y) .
$$

It rests to show that $\kappa(z)$ is a Kummer character in the sense of Definition 14.0.4. Assume that $z<0$. Then $z=(-1) \cdot|z|$. If $l$ is odd then $(-1) \cdot|z|^{1 / l^{n}}$ is a compatible family of $l^{n}$-th roots of $z$, hence $\kappa(z)$ is a Kummer character. If $l=2$ then $e^{2 \pi i / 2 \cdot 2^{n}} \cdot|z|^{1 / 2^{n}}$ is a compatible family of $2^{n}$-th roots of $z$. The associated Kummer character is equal $\kappa(z)+\frac{1}{2}(\chi-1)$, hence $\kappa(z)$ is also a Kummer character. 
14.1. We explain how a path $p$ on $\mathbf{P}_{K}^{1} \backslash\{0, \infty\}$ from $\overrightarrow{01}$ to $\zeta$ determines a compatible family of $l^{n}$-th roots of $\zeta$. Let $z$ be a local parameter at 0 corresponding to the tangential base point $\overrightarrow{01}$. We consider Puiseux elements $\left\{z^{1 / l^{n}}\right\}_{n \in \mathbf{N}}$ such that at 1 they are all equal 1 . By analytic continuation of Puiseux elements first from 1 to $\overrightarrow{01}$ along the canonical path and next along $p$ to $\zeta$ we get a compatible family of $l^{n}$-th roots of $\zeta$. If $\zeta=\overrightarrow{0 \tau}$ is a tangential base point then a path $p$ determines a compatible family of $l^{n}$-th roots of $\tau$. We continue analytically, first from 1 to $\overrightarrow{01}$, next along $p$ to $\zeta$ and finally to $\tau$ along the canonical path from $\overrightarrow{0 \tau}$ to $\tau$.

The corresponding Kummer character we denote by $\kappa(\zeta)_{p}$ to indicate the dependence on the path $p$.

In the next proposition we show that the $l$-adic logarithm $l(\zeta)_{p}$ coincides with the Kummer character $\kappa(\zeta)_{p}$. Let $x$ be a geometric generator of $\pi_{1}\left(\mathbf{P}^{1}(\mathbf{C}) \backslash\{0, \infty\} ; \overrightarrow{01}\right)$. We recall from Part II, Corollary 11.0.7 that the $l$-adic logarithm is defined by the following equality

$$
p^{-1} \cdot \sigma \cdot p \cdot \sigma^{-1}=\mathfrak{f}_{p}(\sigma)=x^{l(\zeta)_{p}(\sigma)} .
$$

Proposition 14.1.0. Let $p$ be a path from $\overrightarrow{01}$ to $\zeta$ on $\mathbf{P}_{K}^{1} \backslash\{0, \infty\}$. Then we have

$$
l(\zeta)_{p}=\kappa(\zeta)_{p} .
$$

If $\zeta=\overrightarrow{0 \tau}$ then $l(\zeta)_{p}=\kappa(\tau)_{t \cdot p}$, where $t$ is a canonical path from $\overrightarrow{0 \tau}$ to $\tau$.

Proof. Let $z$ be a local parameter in 0 corresponding to a tangential base point $\overrightarrow{01}$. The loop $\mathfrak{f}_{p}(\sigma)=p^{-1} \cdot \sigma(p)=p^{-1} \cdot \sigma \cdot p \cdot \sigma^{-1}$ acts on $z^{1 / l^{n}}$ as follows:

$$
\begin{aligned}
z^{1 / l^{n}} \stackrel{\sigma^{-1}}{\longrightarrow} z^{1 / l^{n}} \stackrel{p}{\longrightarrow} \zeta^{1 / l^{n}} & \left(1+\frac{z-\zeta}{\zeta}\right)^{1 / l^{n}} \\
& \stackrel{\sigma}{\longrightarrow} \xi_{l^{n}}^{\kappa(\zeta)_{p}} \zeta^{1 / l^{n}}\left(1+\frac{z-\zeta}{\zeta}\right)^{1 / l^{n}} \stackrel{p^{-1}}{\longrightarrow} \xi_{l^{n}}^{\kappa(\zeta)_{p}} z^{1 / l^{n}} .
\end{aligned}
$$

Now we assume that $\zeta=\overrightarrow{0 \tau}$. Let $t$ be a local parameter corresponding to $\overrightarrow{0 \tau}$. Then we have $z=\tau \cdot t$ and $z^{1 / l^{n}}=\tau^{1 / l^{n}} \cdot t^{1 / l^{n}}\left(z^{1 / l^{n}}\right.$ is real positive over the interval $[0,1]$ and $t^{1 / l^{n}}$ is real positive over the interval $\left.[0, \tau]\right)$. The path $p^{-1} \cdot \sigma \cdot p \cdot \sigma^{-1}$ acts on $z^{1 / l^{n}}$ as follows:

$$
z^{1 / l^{n}} \stackrel{\sigma^{-1}}{\longrightarrow} z^{1 / l^{n}} \stackrel{p}{\longrightarrow} \tau^{1 / l^{n}} \cdot t^{1 / l^{n}} \stackrel{\sigma}{\longrightarrow} \xi_{l^{n}}^{\kappa(\tau)_{p}} \cdot \tau^{1 / l^{n}} \cdot t^{1 / l^{n}} \stackrel{p^{-1}}{\longrightarrow} \xi_{l^{n}}^{\kappa(\tau)_{p}} \cdot z^{1 / l^{n}} .
$$


Proposition 14.1.1. Let $X=\mathbf{P}_{K}^{1} \backslash\left\{a_{1}, \ldots, a_{n}, \infty\right\}$, let $z, v \in \hat{X}(K)$ and let $p$ be a path from $v$ to $z$. Let $g_{i}: X \rightarrow \mathbf{P}_{K}^{1} \backslash\{0, \infty\}$ be given by $g_{i}(z)=z-a_{i}$ for $i=1, \ldots, n$. Let us fix a path $q_{i}$ from $\overrightarrow{01}$ to $g_{i}(v)$ for $i=1, \ldots, n$. (If $v=\overrightarrow{a_{i} a}$ then $g_{i}(v)=\left(a-a_{i}\right) \overrightarrow{01}$ and $g_{j}(v)=a_{i}-a_{j}$ for $i \neq j$.) If

$$
\mathfrak{f}_{p} \equiv x_{1}^{\alpha_{1}} \cdots \cdots x_{n}^{\alpha_{n}} \bmod \Gamma^{2} \pi_{1}\left(X_{\bar{K}} ; v\right)
$$

then

i) if $z, v \in X(K)$ then $\alpha_{i}=\kappa\left(z-a_{i}\right)_{g_{i}(p) \cdot q_{i}}-\kappa\left(v-a_{i}\right)_{q_{i}}$;

ii) if $z \in X(K)$ and $v=\overrightarrow{a_{i} a}$ then $\alpha_{j}=\kappa\left(z-a_{j}\right)_{g_{j}(p) \cdot q_{j}}-\kappa\left(a_{i}-a_{j}\right)_{q_{j}}$ for $i \neq j$ and $\alpha_{i}=\kappa\left(z-a_{i}\right)_{g_{i}(p) \cdot q_{i}}-\kappa\left(a-a_{i}\right)_{q_{i}}$;

iii) if $z=\overrightarrow{a_{j} b}$ and $v=\overrightarrow{a_{i} a}$ then $\alpha_{i}=\kappa\left(a_{j}-a_{i}\right)_{g_{i}(p) \cdot q_{i}}-\kappa\left(a-a_{i}\right)_{q_{i}}$, $\alpha_{j}=\kappa\left(b-a_{j}\right)_{g_{j}(p) \cdot q_{j}}-\kappa\left(a_{i}-a_{j}\right)_{q_{j}}$ and $\alpha_{k}=\kappa\left(a_{j}-a_{k}\right)_{g_{k}(p) \cdot q_{k}}-$ $\kappa\left(a_{i}-a_{k}\right)_{q_{k}}$ for $k \neq i, j$;

iv) if $z=\overrightarrow{a_{i} b}$ and $v=\overrightarrow{a_{i} a}$ then $\alpha_{i}=\kappa\left(b-a_{i}\right)_{g_{i}(p) \cdot q_{i}}-\kappa\left(a-a_{i}\right)_{q_{i}}$ and $\alpha_{j}=0$ for $j \neq i$.

Proof. The proposition follows from Proposition 11.0.17 in Part II and from Proposition 14.1.0.

Observe that we have just proved Theorem F.

14.2. In [S2] and [S3] Soulé has defined certain cohomology classes associated to compatible families of $l$-units. If we take compatible families of cyclotomic $l$-units then we get cyclotomic Soulé classes.

In this subsection we shall discuss relations of Soulé classes with $l$ adic polylogarithms. In $[\mathrm{NW}]$ we have given an arithmetic formula for $l$-adic polylogarithms. We present a proof of this result with some small modifications. (The related result was obtained by O. Gabber a long time ago using different methods.) Next we restrict our attention to the field $K=\mathbf{Q}\left(\mu_{p}\right)$, where $p$ is relatively prime to $l$. We express cyclotomic Soulé classes by $l$-adic polylogarithms evaluated at $p$-th roots of 1 .

Let us choose a compatible family $\left(\xi_{l^{n}}\right)_{n \in \mathbf{N}}$ of primitive $l^{n}$-th roots of 1. Let $p$ be an integer prime to $l$ and let $\xi_{p}$ be a primitive $p$-th root of 1 . Let $\left(\xi_{p}^{1 / l^{n}}\right)_{n \in \mathbf{N}}$ be a family of $p$-th roots of 1 such that $\left(\xi_{p}^{1 / l^{n}}\right)^{l}=\xi_{p}^{1 / l^{n-1}}$, $\left(\xi_{p}^{1 / l^{n}}\right)^{l^{n}}=\xi_{p}$ and for any $n, \xi_{p}^{1 / l^{n}} \in \mu_{p}$. 
Let $q$ be an integer. We set

$u_{m, q}^{n}:=\prod_{k=0}^{l^{n}-1}\left(1-\xi_{l^{n}}^{k} \cdot\left(\xi_{p}^{1 / l^{n}}\right)^{q}\right)^{k^{m}}$ and $v_{m, q}^{n}:=\prod_{k=0}^{l^{n}-1}\left(1-\xi_{l^{n}}^{k} \cdot\left(\xi_{p}^{1 / l^{n}}\right)^{q}\right)^{k^{m}}$

where $n, m \in \mathbf{N}$. One checks that

$$
u_{m, q}^{n}=v_{m, q}^{n} \cdot\left(u_{m, q \cdot l^{-1}}^{n-1}\right)^{l^{m}} .
$$

We define Kummer characters

$$
\kappa_{m, q}^{n}, \bar{\kappa}_{m, q}^{n}: G_{\mathbf{Q}\left(\mu_{l^{n} \cdot p}\right)} \longrightarrow \mathbf{Z} / l^{n}
$$

by

$$
\frac{\sigma\left(\left(u_{m, q}^{n}\right)^{1 / l^{n}}\right)}{\left(u_{m, q}^{n}\right)^{1 / l^{n}}}=\xi_{l^{n}}^{\kappa_{m, q}^{n}(\sigma)} \text { and } \quad \frac{\sigma\left(\left(v_{m, q}^{n}\right)^{1 / l^{n}}\right)}{\left(v_{m, q}^{n}\right)^{1 / l^{n}}}=\xi_{l^{n}}^{\bar{\kappa}_{m, q}^{n}(\sigma)}
$$

The characters $\left(\kappa_{m, q}^{n}\right)_{n \in \mathbf{N}}$ and $\left(\bar{\kappa}_{m, q}^{n}\right)_{n \in \mathbf{N}}$ satisfy

$$
\kappa_{m, q}^{n+1} \equiv \kappa_{m, q}^{n} \bmod l^{n} \quad \text { and } \quad \bar{\kappa}_{m, q}^{n+1} \equiv \bar{\kappa}_{m, q}^{n} \bmod l^{n}
$$

for any $\sigma \in G_{\mathbf{Q}\left(\mu_{l^{n+1} \cdot p}\right)}$. We define

$$
\kappa_{m, q}: G_{\mathbf{Q}\left(\mu_{l} \infty p\right)} \longrightarrow \mathbf{Z}_{l} \quad \text { and } \quad \bar{\kappa}_{m, q}: G_{\mathbf{Q}\left(\mu_{l} \infty, p\right)} \longrightarrow \mathbf{Z}_{l}
$$

by $\kappa_{m, q}(\sigma):=\left(\kappa_{m, q}^{n}(\sigma)\right)_{n \in \mathbf{N}}$ and $\bar{\kappa}_{m, q}(\sigma):=\left(\bar{\kappa}_{m, q}^{n}(\sigma)\right)_{n \in \mathbf{N}}$. It follows from (14.2.1) that

$$
\kappa_{m, q}=\bar{\kappa}_{m, q}+l^{m} \kappa_{m, q \cdot l^{-1}}
$$

Lemma 14.2.3. Let $\sigma \in G_{\mathbf{Q}\left(\mu_{l} \infty . p\right)}$. Then we have

$$
l_{m+1}\left(\xi_{p}^{q}\right)(\sigma)=\frac{(-1)^{m}}{m !} \kappa_{m, q}(\sigma) .
$$

Proof. The lemma follows from $[\mathrm{NW}$. We give a brief sketch of the proof following $[\mathrm{NW}]$.

Let $\pi$ be a path from $\overrightarrow{01}$ to $z$. Let $x$ and $y$ in $\pi_{1}\left(\mathbf{P}_{\overline{\mathbf{Q}}}^{1} \backslash\{0,1, \infty\} ; \overrightarrow{01}\right)$ be standard generators of the fundamental group. Let us set

$$
H_{n}:=\operatorname{ker}\left(\pi_{1}\left(\mathbf{P}_{\overline{\mathbf{Q}}}^{1} \backslash\{0,1, \infty\} ; \overrightarrow{01}\right) \rightarrow \mathbf{Z} / l^{n}\right),
$$


where $x \rightarrow 1$ and $y \rightarrow 0$. The group $H_{n}$ is freely generated by $x^{l^{n}}$ and $x^{i} y x^{-i}\left(i=0, \ldots, l^{n}-1\right)$. Hence we have

$$
\mathfrak{f}_{\pi}(\sigma) \cdot x^{-\kappa_{z}^{0}(\sigma)} \equiv \prod_{i=0}^{l^{n}-1}\left(x^{i} y x^{-i}\right)^{\alpha_{i}^{n}(\sigma)} \bmod \left[H_{n}, H_{n}\right]
$$

for some $\alpha_{0}^{n}(\sigma), \ldots, \alpha_{l^{n}-1}^{n}(\sigma) \in \mathbf{Z}_{l}$. If $m>n$ then $\alpha_{i}^{n}=\sum_{j \equiv i\left(l^{n}\right)} \alpha_{j}^{m}$. Hence we have a measure $d \alpha(\sigma)$ on $\mathbf{Z}_{l}$.

Let us define Kummer characters $\beta_{j, N}^{n}$ as follows

$$
\sigma\left(\left(1-\xi_{l^{n}}^{\left(j-\kappa_{z}^{0}(\sigma)\right) \chi\left(\sigma^{-1}\right)} \cdot z^{1 / l^{n}}\right)^{1 / l^{N}}\right)=\xi_{l^{N}}^{\beta_{j, N}^{n}(\sigma)} \cdot\left(1-\xi_{l^{n}}^{j} \cdot z^{1 / l^{n}}\right)^{1 / l^{N}} .
$$

We have $\beta_{j, M}^{n} \equiv \beta_{j, N}^{n} \bmod l^{N}$ if $M>N$. We set $\beta_{j}^{n}:=\left(\beta_{j, N}^{n}\right)_{N} \in \mathbf{Z}_{l}$. Studying transformations of functions $\left(1-\xi_{l^{n}}^{i} \cdot z^{1 / l^{n}}\right)^{1 / l^{N}}$ along $\mathfrak{f}_{\pi}(\sigma) \cdot x^{-\kappa_{z}^{0}(\sigma)}$ one shows that $\alpha_{j}^{n}=\beta_{j}^{n}$.

We recall that $k: \pi_{1}\left(\mathbf{P}_{\overline{\mathbf{Q}}}^{1} \backslash\{0,1, \infty\} ; \overrightarrow{01}\right) \rightarrow \mathbf{Q}_{l}\{\{X, Y\}\}$ is a continuous multiplicative embedding given by $k(x)=e^{X}$ and $k(y)=e^{Y}$. It follows from (14.2.4) that

$$
\log k\left(\mathfrak{f}_{\pi}(\sigma) \cdot x^{-\kappa_{z}^{0}(\sigma)}\right) \equiv \sum_{k=0}^{\infty} \frac{(-1)^{k}}{k !}\left(\sum_{i=0}^{l^{n}-1} i^{k} \alpha_{i}^{n}(\sigma)\right)\left[Y, X^{k}\right]
$$

modulo logarithms of elements from $k\left(\left[H_{n}, H_{n}\right]\right)$, where $\left[Y, X^{0}\right]:=Y$ and $\left[Y, X^{k}\right]:=\left[\left[Y, X^{k-1}\right], X\right]$ for $k>0$.

Therefore the coefficient at $\left[Y, X^{k}\right]$ of $\log k\left(\mathfrak{f}_{\pi}(\sigma) \cdot x^{-\kappa_{z}^{0}(\sigma)}\right)$ is congruent to $\frac{(-1)^{k}}{k !} \sum_{i=0}^{l^{n}-1} i^{k} \alpha_{i}^{n}(\sigma)$ modulo $l^{n-n_{0}}$, where $n_{0}$ is a positive integer not depending on $n$. Hence this coefficient is equal $\frac{(-1)^{k}}{k !} \int_{\mathbf{z}_{l}} x^{k} d \alpha(\sigma)$.

Let us take $z=\xi_{p}^{q}$ and let $\pi$ be a path from $\overrightarrow{01}$ to $\xi_{p}^{q}$ such that $\kappa_{\xi_{p}^{q}}^{0}(\sigma)=0$ for any $\sigma \in G_{\mathbf{Q}\left(\mu_{p}\right)}$. For such a path $\pi$ the factor $x^{-\kappa_{z}^{0}(\sigma)}$ in the left hand side of (14.2.5) is 1 , hence we get

$$
l_{k+1}\left(\xi_{p}^{q}\right)_{\pi}(\sigma)=\frac{(-1)^{k}}{k !} \int_{\mathbf{Z}_{l}} x^{k} d \alpha(\sigma) .
$$

Observe that $\int_{\mathbf{Z}_{l}} x^{n-1} d \alpha(\sigma) \equiv \sum_{i=0}^{l^{N}-1} i^{n-1} \cdot \alpha_{i}^{N}(\sigma) \bmod l^{N}$. We have $\sum_{i=0}^{l^{N}-1} i^{n-1} \cdot \alpha_{i}^{N}=\sum_{i=0}^{l^{N}-1} i^{n-1} \cdot \beta_{i}^{N}$. Notice that $\sum_{i=0}^{l^{N}-1} i^{n-1} \cdot \beta_{i}^{N} \equiv \kappa_{n-1, q}^{N}$ $\bmod l^{N}$. Hence we get $\int_{\mathbf{Z}_{l}} x^{n-1} d \alpha(\sigma)=\kappa_{n-1, q}(\sigma)$. This implies the lemma. 
14.3. Let $m$ be an integer prime to $l$ and let $K=\mathbf{Q}\left(\mu_{m}\right)$. We recall briefly the construction of the Soulé classes for the field $K$.

Let us set $K_{n}:=K\left(\mu_{l^{n}}\right)$ and $K_{\infty}:=K\left(\mu_{l \infty}\right)$. The extension $K \subset$ $K_{n}$ is unramified outside $l$. Let $M$ be a maximal Galois extension of $K$ unramified outside $l$. Let us set $G:=\operatorname{Gal}(M / K), G_{n}:=\operatorname{Gal}\left(M / K_{n}\right)$, $G_{\infty}:=\operatorname{Gal}\left(M / K_{\infty}\right), \Gamma_{n}:=\operatorname{Gal}\left(K_{n} / K\right)$ and $\Gamma_{\infty}:=\operatorname{Gal}\left(K_{\infty} / K\right)$. The groups $G_{n}$ and $G_{\infty}$ are normal subgroups of $G$ and we have $G / G_{n} \simeq \Gamma_{n}$ and $G / G_{\infty} \simeq \Gamma_{\infty}$. Let us set $\mathcal{R}_{n}:=\mathbf{Z}\left[\frac{1}{l}\right]\left(\mu_{m l^{n}}\right), X_{n}:=\operatorname{Spec} \mathcal{R}_{n}, X_{\infty}:=\lim _{n} X_{n}$ and $X:=\operatorname{Spec} \mathbf{Z}\left[\frac{1}{l}\right]\left(\mu_{m}\right)$.

The exact sequence of sheaves on $X_{n}$

$$
1 \longrightarrow \mu_{l^{n}} \longrightarrow \mathbf{G}_{\mathbf{m}} \stackrel{l^{n}}{\longrightarrow} \mathbf{G}_{\mathbf{m}} \longrightarrow 1
$$

induces a long exact sequence of cohomology

$$
0 \longrightarrow H^{0}\left(X_{n}, \mu_{l^{n}}\right) \longrightarrow H^{0}\left(X_{n}, \mathbf{G}_{\mathbf{m}}\right) \longrightarrow H^{0}\left(X_{n}, \mathbf{G}_{\mathbf{m}}\right) \stackrel{\delta}{\longrightarrow} H^{1}\left(X_{n}, \mu_{l^{n}}\right) \cdots
$$

Let $\left(\xi_{l^{n}}\right)_{n \in \mathbf{N}}$ be a compatible family of primitive $l^{n}$-th roots of 1 as in 14.2. The element $\xi_{l^{n}}$ defines an element in $H^{0}\left(X_{n}, \mu_{l^{n}}\right)$, which we also denote by $\xi_{l^{n}}$. Let $\alpha_{n}:=\xi_{l^{n}} \cup \cdots \cup \xi_{l^{n}}(i-1$ times $)$ and let $u_{n} \in \mathcal{R}_{n}^{*}=H^{0}\left(X_{n} ; \mathbf{G}_{\mathbf{m}}\right)$. Observe that $\alpha_{n} \in H^{0}\left(X_{n}, \mu_{l^{n}}^{\otimes(i-1)}\right)$. Let

$$
N_{n}: H^{1}\left(X_{n}, \mu_{l^{n}}^{\otimes i}\right) \longrightarrow H^{1}\left(X, \mu_{l^{n}}^{\otimes i}\right)
$$

be a transfer map associated to an étale covering $p_{n}: X_{n} \rightarrow X$. Let us set

$$
x_{n}^{i}:=N_{n}\left(\alpha_{n} \cup \delta\left(u_{n}\right)\right) .
$$

LEMMA 14.3.1. Let $\left(u_{n}\right)_{n \in \mathbf{N}}, u_{n} \in \mathcal{R}_{n}^{*}$ be a family of l-units such that $N_{n+1, n}\left(u_{n+1}\right)=u_{n}$, where $N_{n+1, n}: \mathcal{R}_{n+1}^{*} \rightarrow \mathcal{R}_{n}^{*}$ is a norm. Then $r_{n+1, n}\left(x_{n+1}^{i}\right)=x_{n}^{i}$, where $r_{n+1, n}: H^{1}\left(X, \mu_{l^{n+1}}^{\otimes i}\right) \rightarrow H^{1}\left(X, \mu_{l^{n}}^{\otimes i}\right)$ is induced by the projection $\mu_{l^{n+1}} \rightarrow \mu_{l^{n}}$. Hence the family $\left(x_{n}^{i}\right)_{n \in \mathbf{N}}$ defines an element $x^{i}=\left(x_{n}^{i}\right)_{n \in \mathbf{N}} \in H^{1}\left(X, \mathbf{Z}_{l}(i)\right)$.

Proof. One repeats the proof of Lemma 1 in [S2] replacing K-theory by cohomology.

Let us set $u_{n}^{(q)}:=\left(1-\xi_{m}^{q / l^{n}} \cdot \xi_{l^{n}}\right)$. It is clear that the system of $l$ units $\left(u_{n}^{(q)}\right)_{n \in \mathbf{N}}$ is compatible, i.e., it satisfies $N_{n+1, n}\left(u_{n+1}^{(q)}\right)=u_{n}^{(q)}$. Hence 
it defines an element $x^{i}(q) \in H^{1}\left(X, \mathbf{Z}_{l}(i)\right)$. The elements $x^{i}(q)$ are called cyclotomic Soulé elements.

The covering $p_{n}: X_{n} \rightarrow X$ is Galois. It follows from [S1, Lemma 6] that we have a commutative diagram

$$
\begin{aligned}
& \left(\mathcal{R}_{n}^{*} /\left(\mathcal{R}_{n}^{*}\right)^{l^{n}}\right) \otimes \mu_{l^{n}}^{\otimes(i-1)} \stackrel{\delta \otimes i d_{l^{n}}^{\otimes(i-1)}}{\longrightarrow} H^{1}\left(X_{n} ; \mu_{l^{n}}^{\otimes i}\right) \\
& \downarrow N_{\Gamma_{n}} \quad \downarrow p_{n}^{* \circ N_{n}} \\
& \left(\mathcal{R}_{n}^{*} /\left(\mathcal{R}_{n}^{*}\right)^{l^{n}}\right) \otimes \mu_{l^{n}}^{\otimes(i-1)} \stackrel{\delta \otimes i d_{l^{n}}^{\otimes(i-1)}}{\longrightarrow} H^{1}\left(X_{n} ; \mu_{l^{n}}^{\otimes i}\right)
\end{aligned}
$$

where $N_{\Gamma_{n}}(c)=\prod_{\sigma \in \Gamma_{n}} \sigma(c)$. Observe that

$$
\begin{aligned}
& N_{\Gamma_{n}}\left(\left(1-\xi_{m}^{q / l^{n}} \cdot \xi_{l^{n}}\right) \otimes \xi_{l^{n}}^{\otimes(i-1)}\right) \\
& \quad=\prod_{\sigma \in \Gamma_{n}}\left(1-\xi_{m}^{q / l^{n}} \cdot \xi_{l^{n}}^{\chi(\sigma)}\right) \otimes\left(\xi_{l^{n}}^{\chi(\sigma)}\right)^{\otimes(i-1)} \\
& \quad=\prod_{0<k<l^{n},(k, l)=1}\left(1-\xi_{m}^{q / l^{n}} \cdot \xi_{l^{n}}^{k}\right)^{k^{i-1}} \otimes \xi_{l^{n}}^{\otimes(i-1)} .
\end{aligned}
$$

Hence the element

$$
p_{n}^{*}\left(x_{n}^{i}\right) \in H^{1}\left(X_{n} ; \mu_{l^{n}}^{\otimes i}\right) \approx H o m\left(G_{n} ; \mu_{l^{n}}\right) \otimes \mu_{l^{n}}^{\otimes(i-1)}
$$

is given by $\bar{\kappa}_{i-1, q}^{n}$.

We summarize the above discussion in the following proposition.

Proposition 14.3.2. (see also [W2, Lemma 3.3]) The family of l-units $\left(1-\xi_{m}^{q / l^{n}} \cdot \xi_{l^{n}}\right)_{n \in \mathbf{N}}$ defines an element $x^{i}(q)=\left(x_{n}^{i}\right)_{n \in \mathbf{N}} \in{\underset{\lim }{\longleftarrow}}_{n} H^{1}\left(X ; \mu_{l^{n}}^{\otimes i}\right)=$ $H^{1}\left(X ; \mathbf{Z}_{l}(i)\right)$. The restriction of $x_{n}^{i} \in H^{1}\left(X ; \mu_{l^{n}}^{\otimes i}\right)$ to $H^{1}\left(X_{n} ; \mu_{l^{n}}^{\otimes i}\right) \approx$ $\operatorname{Hom}\left(G_{n} ; \mu_{l^{n}}\right) \otimes \mu_{l^{n}}^{\otimes(i-1)}$ is equal to the homomorphism $\bar{\kappa}_{i-1, q}^{n}$, i.e., it is equal to the Kummer character associated to the element $\prod_{0<k<l^{n},\left(k, l^{n}\right)=1}(1-$ $\left.\xi_{m}^{q / l^{n}} \cdot \xi_{l^{n}}^{k}\right)^{k^{i-1}}$.

Corollary 14.3.3. After the restriction to $G_{K\left(\mu_{l} \infty\right)}$ we have the followings identities:

i) $\frac{(-1)^{n}}{n !} x^{n+1}(q)=l_{n+1}\left(\xi_{m}^{q}\right)-l^{n} l_{n+1}\left(\xi_{m}^{q \cdot l^{-1}}\right)$. 
ii) Let $s$ be an order of $l$ in $(\mathbf{Z} / m)^{*}$. Then we have

$$
\left(1-l^{s \cdot n}\right) l_{n+1}\left(\xi_{m}^{q}\right)=\sum_{i=0}^{s-1} \frac{(-1)^{n}}{n !} l^{i \cdot n} x^{n+1}\left(q \cdot l^{-i}\right) .
$$

Proof. The part i) of the corollary follows from (14.2.2), Lemma 14.2.3 and Proposition 14.3.2. The part ii) is an immediate consequence of the part i).

The Soulé classes $x^{n+1}(q)$ and the $l$-adic polylogarithms evaluated at $m$ th roots of 1 are cohomology classes in $H^{1}\left(G_{K} ; \mathbf{Q}_{l}(m+1)\right)$. The restriction map

$H^{1}\left(G_{K} ; \mathbf{Q}(m+1)\right) \rightarrow H^{1}\left(\bigcap_{q=0}^{m-1} H_{m+1}\left(\mathbf{P}_{\mathbf{Q}\left(\mu_{m}\right)}^{1} \backslash\{0,1, \infty\} ; \xi_{m}^{q}, \overrightarrow{01}\right) ; \mathbf{Q}_{l}(m+1)\right)$

is injective by Lemma 3.0.8. Let $d$ be a divisor of $m$. The $l$-adic polylogarithms satisfy the distribution relations

$$
d^{n}\left(\sum_{\xi^{d}=1} l_{n+1}(\xi)\right)=l_{n+1}(1)
$$

and

$$
d^{n}\left(\sum_{\xi^{d}=1} l_{n+1}\left(\xi \cdot \xi_{m}^{j}\right)\right)=l_{n+1}\left(\xi_{m}^{j \cdot d}\right)
$$

for any $j$ such that $0<j<\frac{m}{d}$, the inversion relation

$$
l_{n+1}\left(\xi_{m}^{k}\right)+(-1)^{n+1} l_{n+1}\left(\xi_{m}^{m-k}\right)=0
$$

and the equality

$$
l_{2 n}(\overrightarrow{10})=0
$$

on the subgroup $\bigcap_{q=0}^{m-1} H_{n+1}\left(\mathbf{P}_{\mathbf{Q}\left(\mu_{m}\right)}^{1} \backslash\{0,1, \infty\} ; \xi_{m}^{q}, \overrightarrow{01}\right)$ of $G_{\mathbf{Q}\left(\mu_{m}\right)}$ (see Part II, Section 11.2 and also [W2]).

Hence we get the following corollary.

Corollary 14.3.4. Let $d$ be a divisor of $m$. The cyclotomic Soulé classes satisfy the distribution relations

$$
d^{n}\left(\sum_{i=0}^{d-1} x^{n+1}\left(i \frac{m}{d}\right)\right)=x^{n+1}(m)
$$


and

$$
d^{n}\left(\sum_{i=0}^{d-1} x^{n+1}\left(j+i \frac{m}{d}\right)\right)=x^{n+1}(j \cdot d)
$$

for any $j$ such that $0<j<\frac{m}{d}$, the inversion relation

$$
x^{n+1}(k)+(-1)^{n+1} x^{n+1}(m-k)=0
$$

and the equality

$$
x^{2 n}(0)=0 .
$$

COROLlary 14.3.5. The $\mathbf{Q}_{l}$-vector subspace of $H^{1}\left(G_{K} ; \mathbf{Q}_{l}(n+1)\right)$ generated by the cohomology classes $l_{n+1}\left(\xi_{m}^{k}\right)$ with $0<k<m+1$ is generated by the cohomology classes $l_{n+1}\left(\xi_{m}^{k}\right)$ with $0<k<\frac{m}{2}$ and $(k, m)=1$. This vector space coincides with the $\mathbf{Q}_{l}$-vector subspace of $H^{1}\left(G_{K} ; \mathbf{Q}_{l}(n+1)\right)$ generated by cyclotomic Soulé classes $x^{n+1}(k)$ with $0<k<\frac{m}{2}$ and $(k, m)=1$.

Corollary 14.3.6. The cyclotomic Soulé classes $x^{n+1}(k)$ in $H^{1}\left(G_{K}\right.$; $\left.\mathbf{Q}_{l}(n+1)\right)$ for $0<k<\frac{m}{2}$ and $(k, m)=1$ are linearly independent over $\mathbf{Q}_{l}$ if and only if l-adic polylogarithms $l_{n+1}\left(\xi_{m}^{k}\right)$ for $0<k<\frac{m}{2}$ and $(k, m)=1$ are linearly independent over $\mathbf{Q}_{l}$ in $H^{1}\left(G_{K} ; \mathbf{Q}_{l}(n+1)\right)$.

14.4. We would like to show that the elements $x^{i}(q)$ for $0<q<\frac{m}{2}$ and $(q, m)=1$ are linearly independent and that they generate $H^{1}\left(X ; \mathbf{Z}_{l}(i)\right) \otimes$ Q.

Let us set

$$
E(i-1):=\lim _{N_{n, n-1} \otimes r_{l^{n}}}\left(\mathcal{R}_{n}^{*} \otimes \mu_{l^{n}}^{\otimes(i-1)}\right),
$$

where $N_{n, n-1}: \mathcal{R}_{n}^{*} \rightarrow \mathcal{R}_{n-1}^{*}$ is a norm map and $r_{l^{n}}: \mu_{l^{n}}^{\otimes(i-1)} \rightarrow \mu_{l^{n-1}}^{\otimes(i-1)}$ is a reduction $\bmod l^{n-1}$. The construction in 14.3 which to a compatible family of $l$-units $\left(u_{n}\right)_{n \in \mathbf{N}}$ associates an element $x$ in $H^{1}\left(X ; \mathbf{Z}_{l}(i)\right)$ defines a morphism

$$
\varphi: E(i-1)_{\Gamma_{\infty}} \longrightarrow H^{1}\left(X ; \mathbf{Z}_{l}(i)\right)
$$

such that $\operatorname{ker} \varphi$ and coker $\varphi$ are finite (see [S2, p. 384]).

Let $C(i-1)$ be a subgroup of $E(i-1)$ generated by compatible families of cyclotomic $l$-units. We consider the map

$$
C(i-1)_{\Gamma_{\infty}} \longrightarrow E(i-1)_{\Gamma_{\infty}}
$$

If $K=\mathbf{Q}$ then this map has a finite cokernel (see [S3, Theoreme 3]). 
Observe that the families $\left(\left(1-\xi_{m}^{q / l^{n}} \cdot \xi_{l^{n}}\right) \otimes \xi_{l^{n}}^{\otimes(i-1)}\right)_{n \in \mathbf{N}}$ are in $C(i-1)$. Let us set

$$
u(q):=\left(\left(1-\xi_{m}^{q / l^{n}} \cdot \xi_{l^{n}}\right) \otimes \xi_{l^{n}}^{\otimes(i-1)}\right)_{n \in \mathbf{N}} \cdot
$$

LEMmA 14.4.1. The elements $u(q)\left(0<q<\frac{m}{2}\right.$ and $\left.(q, m)=1\right)$ are linearly independent over $\mathbf{Z}_{l}$ in $C(i-1)_{\Gamma_{\infty}}$. Let $C^{\prime}$ be a $\mathbf{Z}_{l}$-submodule of $C(i-1)_{\Gamma_{\infty}}$ generated by elements $u(q)\left(0<q<\frac{m}{2}\right.$ and $\left.(q, m)=1\right)$. Then the quotient group $C(i-1)_{\Gamma_{\infty}} / C^{\prime}$ is finite.

Proof. Let $C_{n}$ be a subgroup of $\mathbf{Q}\left(\mu_{m l^{n}}\right)^{*}$ generated by $\mu_{m l^{n}}$ and by elements $1-\omega_{n}$, where $\omega_{n} \in \mu_{m l^{n}} \backslash\{1\}$. We recall that

$$
C(i-1):=\lim _{N_{n, n-1} \otimes r_{l^{n}}} C_{n} \otimes \mu_{l^{n}}^{\otimes(i-1)},
$$

where $N_{n, n-1}: C_{n} \rightarrow C_{n-1}$ is a restriction of the norm map $N_{n, n-1}$ : $Q\left(\mu_{m l^{n}}\right)^{*} \rightarrow Q\left(\mu_{m l^{n-1}}\right)^{*}$ and $r_{l^{n}}: \mu_{l^{n}}^{\otimes(i-1)} \rightarrow \mu_{l^{n-1}}^{\otimes(i-1)}$ is a reduction mod $l^{n-1}$. Let $\left(\omega_{n}\right)_{n \in \mathbf{N}}$ be a compatible system of $m l^{n}$-th roots of 1 , i.e., $\omega_{n}=$ $\xi_{m}^{q / l^{n}} \cdot \xi_{l^{n}}^{\alpha}$, where $\alpha \in \mathbf{Z}_{l}$. Assume that $\alpha \in \mathbf{Z}_{l}^{*}$. Then

$$
\left(\left(1-\omega_{n}\right) \otimes \xi_{l^{n}}^{\otimes(i-1)}\right)_{n \in \mathbf{N}}=\frac{1}{\alpha^{i-1}}\left(\left(1-\xi_{m}^{q / l^{n}} \cdot \xi_{l^{n}}\right) \otimes \xi_{l^{n}}^{\otimes(i-1)}\right)_{n \in \mathbf{N}}
$$

in $C(i-1)_{\Gamma_{\infty}}$. Let $k>0$ and $(p, l)=1$. Then the elements $\left(1-\xi_{m}^{q / l^{n}}\right.$. $\left.\xi_{l^{n}}^{p l^{k}}\right) \in C_{n}$ are not in the image of the norm homomorphism $N_{n+1, n}$ : $C_{n+1} \otimes \mathbf{Z} / l^{n+1} \rightarrow C_{n} \otimes \mathbf{Z} / l^{n}$. Observe also that $\xi_{m}^{q / l^{n}} \cdot \xi_{l^{n}} \otimes \xi_{l^{n}}^{\otimes(i-1)}=0$ in $C(i-1) \Gamma_{\infty}$. Hence the elements $u(q):=\left(\left(1-\xi_{m}^{q / l^{n}} \cdot \xi_{l^{n}}\right) \otimes \xi_{l^{n}}^{\otimes(i-1)}\right)_{n \in \mathbf{N}}$ $(m-1 \geq q \geq 0)$ generate $C(i-1)_{\Gamma_{\infty}}$ as a $\mathbf{Z}_{l}$-module.

Let $m=a \cdot b$. Then $\left(1-\xi_{m}^{a k} \cdot \xi_{l^{n}}^{a}\right)=\prod_{\alpha=0}^{a-1}\left(1-\xi_{m}^{k+\alpha b} \cdot \xi_{l^{n}}\right)$. This implies that

$$
\left(1-\xi_{m}^{a k} \cdot \xi_{l^{n}}\right) \otimes \xi_{l^{n}}^{\otimes(i-1)}=a^{i-1}\left(\sum_{\alpha=0}^{a-1}\left(1-\xi_{m}^{k+\alpha b} \cdot \xi_{l^{n}}\right) \otimes \xi_{l^{n}}^{\otimes(i-1)}\right)
$$

in $C(i-1)_{\Gamma_{\infty}}$. Hence we get

$$
u(k a)=a^{i-1} \sum_{\alpha=0}^{a-1} u(k+\alpha b)
$$


in $C(i-1)_{\Gamma_{\infty}}$. The identity

$$
1-\xi_{m}^{q / l^{n}} \cdot \xi_{l^{n}}=-\xi_{m}^{q / l^{n}} \cdot \xi_{l^{n}}\left(1-\xi_{m}^{-q / l^{n}} \cdot \xi_{l^{n}}^{-1}\right)
$$

implies $u(q)=(-1)^{i-1} u(m-q)$. Hence the elements $u(q)\left(0<q<\frac{m}{2}\right.$, $(q, m)=1)$ generate $C(i-1) \Gamma_{\infty}$ modulo torsion.

The projection of $u(q) \in C(i-1)_{\Gamma_{\infty}}$ onto $\left(C_{n} \otimes \mu_{l^{n}}^{\otimes(i-1)}\right)_{\Gamma_{\infty}}$ is equal (1$\left.\xi_{m}^{q / l^{n}} \cdot \xi_{l^{n}}\right) \otimes \xi_{l^{n}}^{\otimes(i-1)}$. It follows from the Bass theorem (see [Wa, Theorem 8.9]) that the elements $\left(1-\xi_{m}^{q / l^{n}} \cdot \xi_{l^{n}}\right) \otimes \xi_{l^{n}}^{\otimes(i-1)}\left(0<q<\frac{m}{2},(q, m)=1\right)$ are linearly independent in $\left(C_{n} \otimes \mu_{l^{n}}^{\otimes(i-1)}\right)_{\Gamma_{\infty}}$.

If the map $C(i-1)_{\Gamma_{\infty}} \rightarrow E(i-1)_{\Gamma_{\infty}}$ has a finite cokernel or finite kernel then Lemma 14.4.1 will imply the following result stated below as a conjecture.

Conjecture 14.4.2. The cyclotomic Soulé classes $x^{n+1}(k)$ in $H^{1}\left(G_{K}\right.$; $\left.\mathbf{Q}_{l}(n+1)\right)$ for $0<k<\frac{m}{2}$ and $(k, m)=1$ are linearly independent over $\mathbf{Q}_{l}$. Consequently $l$-adic polylogarithms $l_{n+1}\left(\xi_{m}^{q}\right)$ for $0<q<\frac{m}{2}$ and $(q, m)=1$ are linearly independent over $\mathbf{Q}_{l}$ in $H^{1}\left(G_{K} ; \mathbf{Q}_{l}(n+1)\right)$ and in $H^{1}\left(\bigcap_{i=0}^{m-1} H_{n+1}\left(\mathbf{P}^{1} \backslash\{0,1, \infty\} ; \xi_{m}^{i}, \overrightarrow{01}\right) ; \mathbf{Q}_{l}\right)$.

The referee pointed to us that the linear independence over $\mathbf{Q}_{l}$ of the cyclotomic Soulé classes $x^{n+1}(k)$ for $0<q<\frac{m}{2}$ and $(q, m)=1$ follows from $[\mathrm{HW}$. However we still did not absorbed the motivic consideration in [HW] so we prefer to left the needed result as a conjecture. It also seems to us that a possible proof of 14.4 .2 should be more elementary without using a motivic machinary.

\section{§15. Applications to Galois actions on fundamental groups}

15.0. We shall use $l$-adic polylogarithms to study the Galois action on the fundamental group of the projective line minus a finite number of points.

Deligne on the conference on polylogarithms in Schloss Ringberg gave a sketch of a proof of his result concerning $\mathbf{P}^{1} \backslash\{0,1,-1, \infty\}$ in the Hodge context (see [D2]). He showed that the Lie algebra associated to the mixed Hodge structures generated by the fundamental group of $\mathbf{P}^{1} \backslash\{0,1,-1, \infty\}$ contains a free Lie algebra on one generator in each odd degree. Using his ideas we are trying to show the analogous result in the Galois setting as well 
as to study some other interesting cases. The first presentation of our results in this direction is in [W1]. In [W3] we study the Hodge analog and there the classical polylogarithms appear. We recall also that the action of $G_{\mathbf{Q}}$ on $\pi_{1}\left(\mathbf{P}_{\overline{\mathbf{Q}}}^{1} \backslash\{0,1, \infty\} ; \overrightarrow{01}\right)$ was studied by Y. Ihara in [I1], [I2] and P. Deligne in [D1]. The $l$-adic polylogarithm $l_{n+1}(\overrightarrow{10})$ in our notation appears in these works (see also [IS]).

Let $K$ be a number field. Let $X=\mathbf{P}_{K}^{1} \backslash\left\{a_{1}, \ldots, a_{n+1}\right\}$ and let $v \in$ $\hat{X}(K)$. Let $x=\left(x_{1}, \ldots, x_{n+1}\right)$ be a sequence of geometric generators of $\pi_{1}(X(\mathbf{C}) ; v)$ associated to a family $\Gamma=\left\{\gamma_{i}\right\}_{i=1, \ldots, n+1}$. Let $\mathbf{X}$ be a set $\left\{X_{1}, \ldots, X_{n}\right\}$. We embed $\pi_{1}\left(X_{\bar{K}} ; v\right)$ into $\mathbf{Q}_{l}\{\{\mathbf{X}\}\}$ mapping $x_{i}$ into $e^{X_{i}}$.

We recall that the action of the Galois group $G_{K}$ on the fundamental group

$$
G_{K} \longrightarrow \operatorname{Aut} \pi_{1}\left(X_{\bar{K}} ; v\right)
$$

induces a Galois representation

$$
\varphi: G_{K} \longrightarrow \operatorname{Aut}\left(\mathbf{Q}_{l}\{\{\mathbf{X}\}\}\right)
$$

and a Lie algebra representation

$$
\operatorname{Lie} \varphi: \operatorname{Lie}\left(G_{1}(X, v) / G_{\infty}(X, v)\right) \otimes \mathbf{Q} \longrightarrow \operatorname{Der}^{*} L(\mathbf{X})
$$

(see Part I, Sections 4.0, 5.0 and 5.1).

Passing with the morphism Lie $\varphi$ to associated graded Lie algebras we get a morphism of associated graded Lie algebras

$$
g r(\operatorname{Lie} \varphi): g r \operatorname{Lie}\left(G_{1}(X, v) / G_{\infty}(X, v)\right) \otimes \mathbf{Q} \longrightarrow \operatorname{Der}^{*} \operatorname{Lie}(\mathbf{X})
$$

Let us define $X_{n+1}$ by the equality

$$
X_{1}+\cdots+X_{n}+X_{n+1}=0 \text {. }
$$

Let us set

$$
\begin{array}{r}
\operatorname{Der}^{* * *} \operatorname{Lie}(\mathbf{X})=\left\{D \in \operatorname{Der} \operatorname{Lie}(\mathbf{X}) \mid \forall k \in\{1, \ldots, n+1\} \exists b_{k} \in \operatorname{Lie}(\mathbf{X})\right. \\
\left.D\left(X_{k}\right)=\left[X_{k}, b_{k}\right]\right\}
\end{array}
$$

Proposition 15.0.1. Let $\sigma \in G_{m}(X, v)$. Then we have

$$
\left(\log \sigma_{x}\right)\left(X_{n+1}\right)=\left[X_{n+1},\left(\log \sigma_{x, \gamma_{n+1}}\right)(1)\right] \bmod \Gamma^{m+2} L(\mathbf{X})
$$


Proof. In the proof we shall omit the subscript $x$ and we shall write $\sigma$ and $\sigma_{\gamma_{n+1}}$ instead of $\sigma_{x}$ and $\sigma_{x, \gamma_{n+1}}$. We have $x_{n+1}=x_{1}^{-1} \cdots x_{n}^{-1}$. Hence $k\left(x_{n+1}\right)=e^{-X_{1}} \cdots e^{-X_{n}}=\exp \left(X_{n+1}+c\right)$, where $c=0 \bmod \Gamma^{2} L(\mathbf{X})$. It follows from Part I, Proposition 2.2.1 that $\sigma\left(X_{n+1}+c\right)=\Lambda_{\gamma_{n+1}}(\sigma)^{-1}$. $\chi(\sigma)\left(X_{n+1}+c\right) \cdot \Lambda_{\gamma_{n+1}}(\sigma)$. Let $\sigma \in G_{1}(X, v)$. One computes that

$$
\begin{aligned}
\left(\log \sigma_{\gamma_{n+1}}\right)(1)= & \left(\Lambda_{\gamma_{n+1}}(\sigma)-1\right) \\
& -\frac{1}{2}\left(\Lambda_{\gamma_{n+1}}(\sigma) \cdot \sigma\left(\Lambda_{\gamma_{n+1}}(\sigma)\right)-2 \Lambda_{\gamma_{n+1}}(\sigma)+1\right)+\frac{1}{3} \cdots .
\end{aligned}
$$

This implies that $\left(\log \sigma_{\gamma_{n+1}}\right)\left(X_{n+1}+c\right)=\left(X_{n+1}+c\right) \cdot\left(\left(\log \sigma_{\gamma_{n+1}}\right)(1)\right)$. It follows from Part I, Proposition 5.1.7 that

$$
(\log \sigma)\left(X_{n+1}+c\right)=\left[X_{n+1}+c,\left(\log \sigma_{\gamma_{n+1}}\right)(1)\right] .
$$

If $\sigma \in G_{m}(X, v)$ then $(\log \sigma)(c)=0 \bmod \Gamma^{m+2} L(\mathbf{X})$ and $\left[c ;\left(\log \sigma_{\gamma_{n+1}}\right)(1)\right]$ $=0 \bmod \Gamma^{m+2} L(\mathbf{X})$. Hence we get

$$
(\log \sigma)\left(X_{n+1}\right)=\left[X_{n+1}, \log \sigma_{\gamma_{n+1}}(1)\right] \bmod \Gamma^{m+2} L(\mathbf{X}) .
$$

Corollary 15.0.2. The image of the homomorphism of Lie algebras

$$
g r(\operatorname{Lie} \varphi): g r \operatorname{Lie}\left(G_{1}(X, v) / G_{\infty}(X, v)\right) \otimes \mathbf{Q} \longrightarrow \operatorname{Der}^{*} \operatorname{Lie}(\mathbf{X})
$$

is contained in $\operatorname{Der}^{* * *} \operatorname{Lie}(\mathbf{X})$.

15.1. In this subsection we shall study the action of the Galois group $G_{\mathbf{Q}\left(\mu_{n}\right)}$ on the fundamental group of $\mathbf{P} \frac{1}{\mathbf{Q}\left(\mu_{n}\right)} \backslash\left\{0, \mu_{n}, \infty\right\}$.

Let us set $V:=\mathbf{P}_{\mathbf{Q}\left(\mu_{n}\right)}^{1} \backslash\left\{0, \mu_{n}, \infty\right\}$. Let us fix an embedding $\overline{\mathbf{Q}} \subset \mathbf{C}$. Let $\xi_{n}=\exp \left(\frac{2 \pi i}{n}\right)$. We chose a tangent vector $\overrightarrow{01}$ as a base point of the fundamental group. At each point $\xi_{n}^{k}$ of the projective line $\mathbf{P}_{\mathbf{Q}\left(\mu_{n}\right)}^{1}$ we choose a tangential base point $v_{k}=\overrightarrow{\xi_{n}^{k}}$. We choose a family $\Gamma=\left\{\gamma_{k}\right\}_{k=0, \ldots, n-1} \cup$ $\left\{\gamma_{\infty}\right\}$ as on Picture 1. The path $\gamma_{k}$ is a path from $\overrightarrow{01}$ to $v_{k}$.

Let $\left(x, y_{0}, \ldots, y_{n-1}, u\right)$ be a sequence of geometric generators of $\pi_{1}(V(\mathbf{C}) ; \overrightarrow{01})$ corresponding to the family $\Gamma$. We have

$$
y_{n-1} \cdot y_{n-2} \cdots \cdot y_{1} \cdot y_{0} \cdot u \cdot x=1 .
$$

Let $f_{k}: V \rightarrow V$ be given by $f_{k}(z)=\xi_{n}^{k} \cdot z$. Then

$$
\left(f_{k}\right)_{*} \mathfrak{f}_{\gamma_{0}}(\sigma)=\mathfrak{f}_{f_{k}\left(\gamma_{0}\right)}(\sigma) .
$$




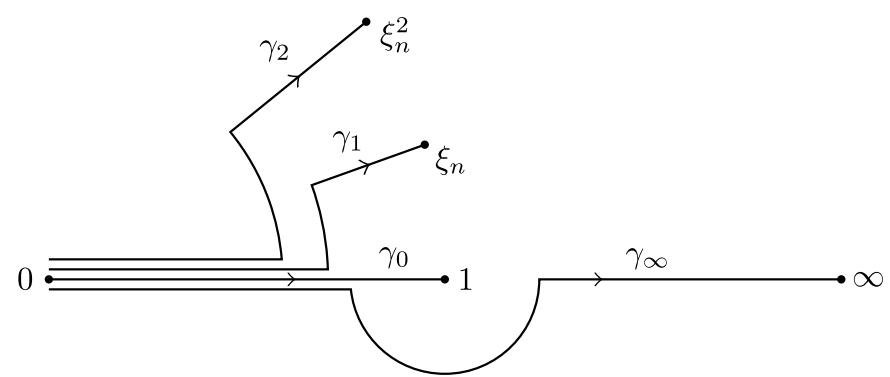

Picture 1

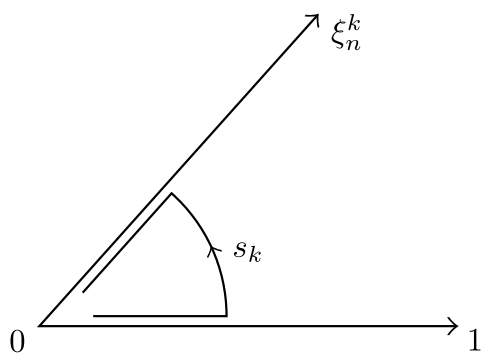

Picture 2

Observe that $f_{k}\left(\gamma_{0}\right)$ is a path (an interval) from $\overrightarrow{0 \xi_{n}^{k}}$ to $\overrightarrow{\xi_{n}^{k}}$. Let $s_{k}$ be a path from $\overrightarrow{01}$ to $\overrightarrow{0 \xi_{n}^{k}}$ as on Picture 2 .

We have

$$
s_{k}^{-1} \cdot\left(f_{k}\right)_{*}(x) \cdot s_{k}=x, s_{k}^{-1} \cdot\left(f_{k}\right)_{*}\left(y_{l}\right) \cdot s_{k}=y_{l+k} \quad \text { if } k+l<n
$$

and

$$
s_{k}^{-1} \cdot\left(f_{k}\right)_{*}\left(y_{l}\right) \cdot s_{k}=x^{-1} \cdot y_{l+k-n} \cdot x \quad \text { if } \quad k+l>n-1 .
$$

It follows from (15.1.2) that

$$
\begin{aligned}
s_{k}^{-1} & \cdot\left(f_{k}\right)_{*}\left(\mathfrak{f}_{\gamma_{0}}(\sigma)\left(x, y_{0}, \ldots, y_{n-1}\right)\right) \cdot s_{k} \\
& =\mathfrak{f}_{\gamma_{0}}(\sigma)\left(x, y_{k}, \ldots, y_{n-1}, x^{-1} \cdot y_{0} \cdot x, \ldots, x^{-1} \cdot y_{k-1} \cdot x\right)
\end{aligned}
$$

for $k=0,1, \ldots, n-1$. Observe that $\gamma_{k}=f_{k}\left(\gamma_{0}\right) \cdot s_{k}$. It follows from Part I, Lemma 1.0.6 and the equality $\mathfrak{f}_{s_{k}}(\sigma)=x^{\frac{k}{n}(\chi(\sigma)-1)}$ that

$$
\mathfrak{f}_{\gamma_{k}}(\sigma)=s_{k}^{-1} \cdot \mathfrak{f}_{f_{k}\left(\gamma_{0}\right)}(\sigma) \cdot s_{k} \cdot x^{\frac{k}{n}(\chi(\sigma)-1)} .
$$


It rests to calculate $\sigma(u)$. Let $g: V \rightarrow V$ be given by $g(z)=\frac{1}{z}$ and let $\delta:=g\left(\gamma_{0}^{-1}\right)$. Observe that $\gamma_{\infty}=\delta \cdot t \cdot \gamma_{0}$, where $t$ is a path from $\frac{z}{10}$ to $\overrightarrow{1 \infty}$ as on Picture 3.

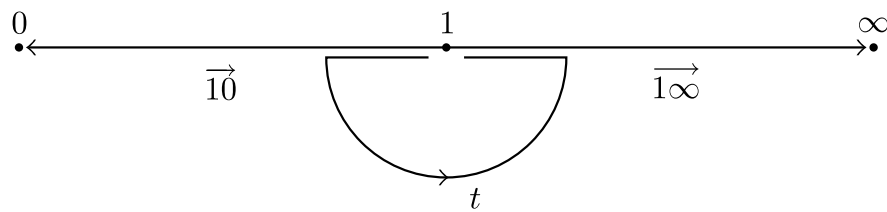

Picture 3

One checks that

$$
\mathfrak{f}_{\gamma_{\infty}}=\gamma_{0}^{-1} \cdot t^{-1} \cdot g_{*}\left(\gamma_{0} \cdot\left(\mathfrak{f}_{\gamma_{0}}\right)^{-1} \cdot \gamma_{0}^{-1}\right) \cdot t \cdot \gamma_{0} \cdot y_{0}^{\frac{\chi-1}{2}} \cdot \mathfrak{f}_{\gamma_{0}} .
$$

Observe that

$$
\begin{aligned}
& \gamma_{0}^{-1} \cdot t^{-1} \cdot g_{*}\left(\gamma_{0} \cdot x \cdot \gamma_{0}^{-1}\right) \cdot t \cdot \gamma_{0}=u \\
& \gamma_{0}^{-1} \cdot t^{-1} \cdot g_{*}\left(\gamma_{0} \cdot y_{0} \cdot \gamma_{0}^{-1}\right) \cdot t \cdot \gamma_{0}=y_{0}
\end{aligned}
$$

and

$$
\begin{aligned}
& \gamma_{0}^{-1} \cdot t^{-1} \cdot g_{*}\left(\gamma_{0} \cdot y_{k} \cdot \gamma_{0}^{-1}\right) \cdot t \cdot \gamma_{0} \\
& \quad=x \cdot y_{n-1} \cdots \cdot y_{n-k+1} \cdot y_{n-k} \cdot y_{n-k+1}^{-1} \cdots \cdot y_{n-1}^{-1} \cdot x^{-1}
\end{aligned}
$$

for $k=1, \ldots, n-1$.

Proposition 15.1.7. The action of $G_{\mathbf{Q}\left(\mu_{n}\right)}$ on $\pi_{1}\left(\mathbf{P}_{\overline{\mathbf{Q}}}^{1} \backslash\left\{0, \mu_{n}, \infty\right\} ; \overrightarrow{01}\right)$ is given by

$$
\begin{gathered}
\sigma(x)=x^{\chi(\sigma)}, \\
\sigma\left(y_{k}\right)=x^{-\frac{k}{n}(\chi(\sigma)-1)} \cdot \mathfrak{f}_{\gamma_{0}}(\sigma)\left(x, y_{k}, \ldots, y_{n-1}, x^{-1} \cdot y_{0} \cdot x, \ldots, x^{-1} \cdot y_{k-1} \cdot x\right)^{-1} \\
\cdot y_{k}^{\chi(\sigma)} \cdot \mathfrak{f}_{\gamma_{0}}(\sigma)\left(x, y_{k}, \ldots, y_{n-1}, x^{-1} \cdot y_{0} \cdot x, \ldots, x^{-1} \cdot y_{k-1} \cdot x\right) \cdot x^{\frac{k}{n}}(\chi(\sigma)-1)
\end{gathered}
$$

for $k=0, \ldots, n-1$ and

$$
\begin{aligned}
& \sigma(u)=\mathfrak{f}_{\gamma_{0}}(\sigma)\left(x, y_{0}, \ldots, y_{n-1}\right)^{-1} \cdot y_{0}^{\frac{1-\chi(\sigma)}{2}} \\
& \quad \cdot \mathfrak{f}_{\gamma_{0}}(\sigma)\left(u, y_{0}, x \cdot y_{n-1} \cdot x^{-1}, \ldots, x \cdot y_{n-1} \cdots y_{2} \cdot y_{1} \cdot y_{2}^{-1} \cdots y_{n-1}^{-1} \cdot x^{-1}\right) \cdot u^{\chi(\sigma)} \\
& \quad \cdot \mathfrak{f}_{\gamma_{0}}(\sigma)\left(u, y_{0}, x \cdot y_{n-1} \cdot x^{-1}, \ldots, x \cdot y_{n-1} \cdots y_{2} \cdot y_{1} \cdot y_{2}^{-1} \cdots y_{n-1}^{-1} \cdot x^{-1}\right)^{-1} \\
& \quad \cdot y_{0}^{\frac{\chi(\sigma)-1}{2}} \cdot \mathfrak{f}_{\gamma_{0}}(\sigma)\left(x, y_{0}, \ldots, y_{n-1}\right) .
\end{aligned}
$$


Proof. It follows follows from Part I, Proposition 2.2.1 and from the equalities (15.1.1)-(15.1.6) that $\sigma(x), \sigma\left(y_{k}\right)$ and $\sigma(u)$ are given by the formulas of the proposition.

15.2. Let us set $\mathbf{V}:=\left\{X, Y_{0}, \ldots, Y_{n-1}\right\}$. Let

$$
k: \pi_{1}\left(P \frac{1}{\mathbf{Q}\left(\mu_{n}\right)} \backslash\left\{0, \mu_{n}, \infty\right\} ; \overrightarrow{01}\right) \longrightarrow \mathbf{Q}_{l}\{\{\mathbf{V}\}\}
$$

be a continuous multiplicative embedding given by $k(x)=e^{X}$ and $k\left(y_{j}\right)=$ $e^{Y_{i}}$ for $j=0, \ldots, n-1$. We denote by $\operatorname{Lie}(\mathbf{V})(\operatorname{resp} . L(\mathbf{V}))$ a free Lie algebra (resp. a completed free Lie algebra) over $\mathbf{Q}_{l}$ on the set $\mathbf{V}$. We define $U$ by the equality $X+Y_{0}+\cdots+Y_{n-1}+U=0$. We shall study elements $\Lambda_{\gamma_{k}}(\sigma)\left(X, Y_{0}, \ldots, Y_{n-1}\right)$.

Lemma 15.2.1. Let $\sigma \in G_{m}(V, \overrightarrow{01})$. Then we have

$$
\begin{aligned}
& \log \left(\Lambda_{\gamma_{k}}(\sigma)\left(X, Y_{0}, \ldots, Y_{n-1}\right)\right) \\
& \quad \equiv \log \left(\Lambda_{\gamma_{0}}(\sigma)\left(X, Y_{k}, \ldots, Y_{n-1}, Y_{0}, \ldots, Y_{k-1}\right)\right) \bmod \Gamma^{m+1} L(\mathbf{V}) .
\end{aligned}
$$

Proof. We recall that $\Lambda_{\gamma_{0}}(\sigma)\left(X, Y_{0}, \ldots, Y_{n-1}\right)=k\left(\mathfrak{f}_{\gamma_{0}}(\sigma)\left(x, y_{0}, \ldots\right.\right.$, $\left.\left.y_{n-1}\right)\right)$ and $\Lambda_{\gamma_{k}}(\sigma)\left(X, Y_{0}, \ldots, Y_{n-1}\right)=k\left(\mathfrak{f}_{\gamma_{k}}(\sigma)\left(x, y_{0}, \ldots, y_{n-1}\right)\right)$. It follows from (15.1.4), (15.1.1) and (15.1.3) that

$$
\begin{aligned}
& \Lambda_{\gamma_{k}}(\sigma)\left(X, Y_{0}, \ldots, Y_{n-1}\right) \\
& \quad=k\left(\mathfrak{f}_{\gamma_{0}}(\sigma)\left(x, y_{k}, \ldots, y_{n-1}, x^{-1} \cdot y_{0} \cdot x, \ldots, x^{-1} \cdot y_{k-1} \cdot x\right) \cdot x^{\frac{k}{n}(\chi(\sigma)-1)}\right) .
\end{aligned}
$$

Hence for $\sigma \in G_{1}(V, \overrightarrow{01})$ we have

$$
\begin{aligned}
& \Lambda_{\gamma_{k}}(\sigma)\left(X, Y_{0}, \ldots, Y_{n-1}\right)=\Lambda_{\gamma_{0}}(\sigma)\left(X, Y_{k}, \ldots, Y_{n-1}\right. \\
& \left.Y_{0}+\sum_{p=1}^{\infty} \frac{1}{p !}\left[\left[Y_{0}, X\right] X^{p-1}\right], \ldots, Y_{k-1}+\sum_{p=1}^{\infty} \frac{1}{p !}\left[\left[Y_{k-1}, X\right] X^{p-1}\right]\right) .
\end{aligned}
$$

If $\sigma \in G_{m}(V, \overrightarrow{01})$ then $\log \left(\Lambda_{\gamma_{k}}(\sigma)\left(X, Y_{0}, \ldots, Y_{n-1}\right)\right) \equiv 0 \bmod \Gamma^{m} L(\mathbf{V})$. Hence $\log \left(\Lambda_{\gamma_{k}}(\sigma)\left(X, Y_{0}, \ldots, Y_{n-1}\right)\right) \equiv \log \left(\Lambda_{\gamma_{0}}(\sigma)\left(X, Y_{k}, \ldots, Y_{n-1}, Y_{0}, \ldots\right.\right.$, $\left.\left.Y_{k-1}\right)\right) \bmod \Gamma^{m+1} L(\mathbf{V})$ for any $\sigma \in G_{m}(V, \overrightarrow{01})$.

Lemma 15.2.2. Let $\sigma \in G_{m}(V, \overrightarrow{01})$. Then we have $\left(\log \sigma_{\gamma_{k}}\right)(1) \equiv \log \left(\Lambda_{\gamma_{0}}(\sigma)\left(X, Y_{k}, \ldots, Y_{n-1}, Y_{0}, \ldots, Y_{k-1}\right)\right) \bmod \Gamma^{m+1} L(\mathbf{V})$. 
Proof. Let $w \in \mathbf{Q}_{l}\{\{\mathbf{V}\}\}$. We recall that $\sigma_{\gamma_{k}}(w)=\Lambda_{\gamma_{k}}(\sigma)\left(X, Y_{0}, \ldots\right.$, $\left.Y_{n-1}\right) \cdot \sigma(w)$. Hence $\log \sigma_{\gamma_{k}}=L_{\log \left(\Lambda_{\gamma_{k}}(\sigma)\left(X, Y_{0}, \ldots, Y_{n-1}\right)\right)} \bigcirc \log \sigma$, where $\bigcirc$ is the $\mathrm{BCH}$ multiplication. We have

$$
\begin{aligned}
\log \sigma_{\gamma_{k}}=L_{\log \left(\Lambda_{\gamma_{k}}(\sigma)\left(X, Y_{0}, \ldots, Y_{n-1}\right)\right)}+\log \sigma \\
\quad+\frac{1}{2} L_{-(\log \sigma)\left(\log \left(\Lambda_{\gamma_{k}}(\sigma)\left(X, Y_{0}, \ldots, Y_{n-1}\right)\right)\right)}+\cdots .
\end{aligned}
$$

Observe that $\log \left(\Lambda_{\gamma_{k}}(\sigma)\left(X, Y_{0}, \ldots, Y_{n-1}\right)\right)=0 \bmod \Gamma^{m} L(\mathbf{V})$. Hence

$$
(\log \sigma)\left(\log \left(\Lambda_{\gamma_{k}}(\sigma)\left(X, Y_{0}, \ldots, Y_{n-1}\right)\right)\right)=0 \bmod \Gamma^{m+1} L(\mathbf{V}) .
$$

This implies that the term $-(\log \sigma)\left(\log \left(\Lambda_{\gamma_{k}}(\sigma)\left(X, Y_{0}, \ldots, Y_{n-1}\right)\right)\right)$ vanishes $\bmod \Gamma^{m+1} L(\mathbf{V})$. One shows that other terms also vanish $\bmod \Gamma^{m+1} L(\mathbf{V})$ except the first two terms. This implies that

$$
\left(\log \sigma_{\gamma_{k}}\right)(1) \equiv \log \left(\Lambda_{\gamma_{k}}(\sigma)\left(X, Y_{0}, \ldots, Y_{n-1}\right)\right) \bmod \Gamma^{m+1} L(\mathbf{V}) .
$$

Now the lemma follows from Lemma 15.2.1.

We recall that we have a morphism of Lie algebras

$$
\operatorname{Lie} \varphi: \operatorname{Lie}\left(G_{1}(V, \overrightarrow{01}) / G_{\infty}(V, \overrightarrow{01})\right) \otimes \mathbf{Q} \longrightarrow \operatorname{Der}^{*} L(\mathbf{V})
$$

and a morphism of associated graded Lie algebras

$$
g r(\operatorname{Lie} \varphi): g r \operatorname{Lie}\left(G_{1}(V, \overrightarrow{01}) / G_{\infty}(V, \overrightarrow{01})\right) \otimes \mathbf{Q} \longrightarrow \operatorname{Der}{ }^{*} \operatorname{Lie}(\mathbf{V}) .
$$

Proposition 15.2.3. For any $\sigma \in \operatorname{gr} \operatorname{Lie}\left(G_{1}(V, \overrightarrow{01}) / G_{\infty}(V, \overrightarrow{01})\right)$ we have $\operatorname{gr}(\operatorname{Lie} \varphi)(\sigma)(X)=0$ and

$$
\operatorname{gr}(\operatorname{Lie} \varphi)(\sigma)\left(Y_{k}\right)=\left[Y_{k}, \lambda(\sigma)\left(X, Y_{k}, \ldots, Y_{n-1}, Y_{0}, \ldots, Y_{k-1}\right)\right]
$$

$(k=0,1, \ldots, n-1)$ for some element $\lambda(\sigma)\left(X, Y_{0}, \ldots, Y_{n-1}\right) \in \operatorname{Lie}(\mathbf{V})$.

Proof. If $\sigma \in G_{1}(V, \overrightarrow{01})$ then $\log \sigma$ is a derivation of the Lie algebra $L(\mathbf{V})$ such that $(\log \sigma)(X)=0$ and $(\log \sigma)\left(Y_{k}\right)=\left[Y_{k},\left(\log \sigma_{\gamma_{k}}\right)(1)\right]$ for $k=$ $0, \ldots, n-1$ (see Part I, Proposition 5.1.8). Hence the proposition follows from Lemma 15.2.2.

DeFinition 15.2.4. We set

$$
\begin{aligned}
& \operatorname{Der}_{\mathbf{Z} / n}^{*} \operatorname{Lie}(\mathbf{V}):=\left\{D \in \operatorname{Der}^{*} \operatorname{Lie}(\mathbf{V}) \mid\right. \\
& \quad \exists \beta\left(X, Y_{0}, \ldots, Y_{n-1}\right) \in \operatorname{Lie}(\mathbf{V}) \forall k \in\{0, \ldots, n-1\}, \\
& \left.D(X)=0 \text { and } D\left(Y_{k}\right)=\left[Y_{k}, \beta\left(X, Y_{k}, \ldots, Y_{n-1}, Y_{0}, \ldots, Y_{k-1}\right)\right]\right\} .
\end{aligned}
$$


LEMMA 15.2.5. The image of the homomorphism gr $($ Lie $\varphi)$ is contained in $\operatorname{Der}_{\mathbf{Z} / n}^{*} \operatorname{Lie}(\mathbf{V})$.

Proof. It follows from Proposition 15.2.3 that the image of $g r(\operatorname{Lie} \varphi)$ is contained in $\operatorname{Der}_{\mathbf{Z} / n}^{*} \operatorname{Lie}(\mathbf{V})$.

DeFinition 15.2.6. We set

$$
\begin{aligned}
& \operatorname{Der}_{\mathbf{Z} / n}^{*, \infty} \operatorname{Lie}(\mathbf{V})=\left\{D \in \operatorname{Der}^{*} \operatorname{Lie}(\mathbf{V}) \mid\right. \\
& \quad \exists \beta\left(X, Y_{0}, \ldots, Y_{n-1}\right) \in \operatorname{Lie}(\mathbf{V}) \forall k \in\{0, \ldots, n-1\}, \\
& D(X)=0, D\left(Y_{k}\right)=\left[Y_{k}, \beta\left(X, Y_{k}, \ldots, Y_{n-1}, Y_{0}, \ldots, Y_{k-1}\right)\right] \text { and } \\
& \left.D(U)=\left[U,-\beta\left(U, Y_{0}, Y_{n-1}, \ldots, Y_{1}\right)+\beta\left(X, Y_{0}, \ldots, Y_{n-1}\right)\right]\right\} .
\end{aligned}
$$

LEMMA 15.2.7. The image of the homomorphism $\operatorname{gr}(\operatorname{Lie} \varphi)$ is contained in $\operatorname{Der}_{\mathbf{Z} / n}^{*, \infty} \operatorname{Lie}(\mathbf{V})$.

Proof. The lemma follows from the formula for $\sigma(u)$ in Proposition 15.1.7. The detail proof requires analogs of Lemmas 15.2.1 and 15.2.2 for $\gamma_{\infty}$. We left details to the reader.

We denote by $\operatorname{Im}(g r(\operatorname{Lie} \varphi))$ the image of the morphism

$$
g r(\operatorname{Lie} \varphi): g r \operatorname{Lie}\left(G_{1}(V, \overrightarrow{01}) / G_{\infty}(V, \overrightarrow{01})\right) \otimes \mathbf{Q} \longrightarrow \operatorname{Der}_{\mathbf{Z} / n}^{*} \operatorname{Lie}(\mathbf{V})
$$

Observe that $\operatorname{Im}(g r(\operatorname{Lie} \varphi))$ is a Lie subalgebra of $\operatorname{Der}_{\mathbf{Z} / n}^{*} \operatorname{Lie}(\mathbf{V})$.

The derivation $D \in \operatorname{Der}_{\mathbf{Z} / n}^{*} \operatorname{Lie}(\mathbf{V})$ such that $D\left(Y_{0}\right)=\left[Y_{0}, \beta\right]$ we denote by $D_{\beta}$. Observe that $\operatorname{Der}_{\mathbf{Z} / n}^{*} \operatorname{Lie}(\mathbf{V}) \approx \operatorname{Lie}(\mathbf{V}) /\left\langle Y_{0}\right\rangle$ as vector spaces. We introduce a new bracket \{\} on $\operatorname{Lie}(\mathbf{V})$ by setting

$$
\left\{\beta, \beta^{\prime}\right\}:=\left[\beta, \beta^{\prime}\right]+D_{\beta}\left(\beta^{\prime}\right)-D_{\beta^{\prime}}(\beta) .
$$

This new Lie algebra we denote by $(\operatorname{Lie}(\mathbf{V}),\{\})$. Observe that $\left\langle Y_{0}\right\rangle$ is a Lie ideal of $(\operatorname{Lie}(\mathbf{V}),\{\})$. Hence we can form a quotient Lie algebra which we denote by $\left(\operatorname{Lie}(\mathbf{V}) /\left\langle Y_{0}\right\rangle,\{\}\right)$.

LEMmA 15.2.8. The Lie algebras $\operatorname{Der}_{\mathbf{Z} / n}^{*} \operatorname{Lie}(\mathbf{V})$ and $\left(\operatorname{Lie}(\mathbf{V}) /\left\langle Y_{0}\right\rangle,\{\}\right)$ are isomorphic.

Proof. The isomorphism associates to $D_{\beta}$ the class of $\beta$ in $\operatorname{Lie}(\mathbf{V}) /\left\langle Y_{0}\right\rangle$. 
Definition 15.2.9. We denote by $I_{k}$ a Lie ideal of Lie( $\left.\mathbf{V}\right)$ generated by Lie brackets which contain at least $k$ elements (with repetitions) among $Y_{0}, Y_{1}, \ldots, Y_{n-1}$.

Let $\mathcal{S}$ be a subset of $\{0, \ldots, n-1\}$. We denote by $I_{r}(\mathcal{S})$ a Lie ideal of Lie $(\mathbf{V})$ generated by Lie brackets which contain at least $r$ elements (with repetitions) among $Y_{0}, Y_{1}, \ldots, Y_{n-1}$ and at least one of these elements is $Y_{k}$ with $k \in \mathcal{S}$ and at least one of these elements is $Y_{j}$ with $j \notin \mathcal{S}$.

We denote by $A(\mathcal{S})$ a Lie subalgebra of Lie $(\mathbf{V})$ generated by elements $Y_{k}(k \in \mathcal{S})$, and by $A(\mathcal{S})_{r}$ the degree $r$ part of $A(\mathcal{S})$.

Recall that $\Gamma^{m+1} \operatorname{Lie}(\mathbf{V})$ is the $(m+1)$-st component of the lower central series of the Lie algebra $\operatorname{Lie}(\mathbf{V})$. In the following discussions, we often look at elements of $\operatorname{Lie}(\mathbf{V})$ modulo the Lie ideal $I_{k}+\Gamma^{m+1} \operatorname{Lie}(\mathbf{V})$.

LEMMA 15.2.10. Let $\mathcal{S}$ be a subset of $\{0, \ldots, n-1\}$ such that $(\mathcal{S}+\mathcal{S}) \cap$ $\mathcal{S}=\emptyset$ (the sum of two elements of $\mathcal{S}$ is calculated $\bmod n)$. Let $w \in I_{r}(\mathcal{S})$, $w^{\prime} \in I_{r^{\prime}}(\mathcal{S})$ and $a \in A(\mathcal{S})_{t}$. Then $D_{a}(w) \in I_{r+t}(\mathcal{S}), D_{w}(a) \in I_{r+t}(\mathcal{S})$ and $D_{w}\left(w^{\prime}\right) \in I_{r+r^{\prime}}(\mathcal{S})$.

15.3. We shall calculate coefficients of $\log \left(\Lambda_{\gamma_{k}}(\sigma)\left(X, Y_{0}, \ldots, Y_{n-1}\right)\right)$. We shall show that they are expressed by $l$-adic polylogarithms evaluated at elements of $\mu_{n}$.

Lemma 15.3.1. Let $\sigma \in G_{m}(V, \overrightarrow{01})$. If $m>1$ then

$$
\begin{array}{r}
\log \left(\Lambda_{\gamma_{0}}(\sigma)\left(X, Y_{0}, \ldots, Y_{n-1}\right)\right) \equiv \sum_{k=0}^{n-1} l_{m}\left(\xi_{n}^{n-k}\right)(\sigma)\left[\left[Y_{k}, X\right] X^{m-2}\right] \\
\bmod I_{2}+\Gamma^{m+1} L(\mathbf{V})
\end{array}
$$

If $m=1$ then

$$
\log \left(\Lambda_{\gamma_{0}}(\sigma)\left(X, Y_{0}, \ldots, Y_{n-1}\right)\right) \equiv \sum_{k=1}^{n-1} l\left(1-\xi_{n}^{n-k}\right)(\sigma) Y_{k} \quad \bmod \Gamma^{2} L(\mathbf{V})
$$

Proof. It follows from the definition of $l$-adic polylogarithms that the coefficient of $\log \left(\Lambda_{\gamma_{0}}(\sigma)\left(X, Y_{0}, \ldots, Y_{n-1}\right)\right)$ at $\left[\left[Y_{0}, X\right] X^{m-2}\right]$ is $l_{m}(\overrightarrow{10})(\sigma)$ and the coefficient of $\log \left(\Lambda_{\gamma_{k}}(\sigma)\left(X, Y_{0}, \ldots, Y_{n-1}\right)\right)$ at $\left[\left[Y_{0}, X\right] X^{m-2}\right]$ is $l_{m}\left(\xi_{n}^{k}\right)(\sigma)$ 
for $0<k<n$. It follows from Lemma 15.2.1 that for $\sigma \in G_{m}(V, \overrightarrow{01})$

$$
\begin{array}{r}
\log \left(\Lambda_{\gamma_{0}}(\sigma)\left(X, Y_{0}, \ldots, Y_{n-1}\right)\right) \equiv \sum_{k=0}^{n-1} l_{m}\left(\xi_{n}^{n-k}\right)(\sigma)\left[\left[Y_{k}, X\right] X^{m-2}\right] \\
\bmod I_{2}+\Gamma^{m+1} L(\mathbf{V})
\end{array}
$$

If $m=1$ then the coefficient of $\log \left(\Lambda_{\gamma_{0}}(\sigma)\left(X, Y_{0}, \ldots, Y_{n-1}\right)\right)$ at $Y_{0}$ vanishes and the coefficient of $\log \left(\Lambda_{\gamma_{k}}(\sigma)\left(X, Y_{0}, \ldots, Y_{n-1}\right)\right)$ at $Y_{0}$ is $l_{1}\left(\xi_{n}^{k}\right)$. To finish the proof we observe that $l_{1}(z)=l(1-z)$ on $G_{1}(V, \overrightarrow{01})$.

Lemma 15.3.2. Let $\sigma \in G_{m}(V, \overrightarrow{01})$. Then

$$
\begin{array}{r}
\operatorname{gr}(\operatorname{Lie} \varphi)(\sigma)\left(Y_{0}\right) \equiv\left[Y_{0}, \sum_{k=0}^{n-1} l_{m}\left(\xi_{n}^{n-k}\right)(\sigma)\left[\left[Y_{k}, X\right] X^{m-2}\right]\right] \\
\bmod I_{3}+\Gamma^{m+2} L(\mathbf{V})
\end{array}
$$

for $m>1$. If $m=1$ then $\operatorname{gr}(\operatorname{Lie} \varphi)(\sigma)\left(Y_{0}\right) \equiv\left[Y_{0}, \sum_{k=1}^{n-1} l\left(1-\xi_{n}^{n-k}\right) Y_{k}\right]$ $\bmod \Gamma^{3} L(\mathbf{V})$.

Proof. The lemma follows from Part I, Proposition 5.1.8 and from Lemmas 15.2.2 and 15.3.1.

15.4. We shall study the image of the homomorphism of Lie algebras

$$
g r(\operatorname{Lie} \varphi): g r \operatorname{Lie}\left(G_{1}(V, \overrightarrow{01}) / G_{\infty}(V, \overrightarrow{01})\right) \otimes \mathbf{Q} \longrightarrow \operatorname{Der}_{\mathbf{Z} / n}^{*} \operatorname{Lie}(\mathbf{V})
$$

First we assume that $n$ is a prime number and for traditional reason we shall denote it by $p$. Hence $V=\mathbf{P}_{\mathbf{Q}\left(\mu_{p}\right)}^{1} \backslash\left\{0, \mu_{p}, \infty\right\}$. We recall that fundamental groups considered in this paper are pro-finite $l$-groups. Now we shall use Conjecture 14.4.2. We shall assume that $p$ is different from $l$. We have not express in this paper $l$-adic polylogarithms evaluated at $l$-th roots of 1 by Soulé classes, hence we have to suppose that $p \neq l$.

Proposition 15.4.1. Let $p$ be a prime number greater than 2 and different from l. Assume that for each $m>1$ the cyclotomic Soulé classes $x^{m}(k)$ in $H^{1}\left(G_{\mathbf{Q}\left(\mu_{p}\right)} ; \mathbf{Q}_{l}(m)\right)$ for $k=1,2, \ldots, \frac{p-1}{2}$ are linearly independent over $\mathbf{Q}_{l}$. Then there are elements $\sigma_{1}^{k}, \sigma_{2}^{k}, \ldots, \sigma_{n}^{k}, \ldots\left(k=1, \ldots, \frac{p-1}{2}\right)$ in 
$g r \operatorname{Lie}\left(G_{1}(V, \overrightarrow{01}) / G_{\infty}(V, \overrightarrow{01})\right) \otimes \mathbf{Q}$ such that any $\sigma_{i}^{k}$ is homogenous of degree $i$ and

$$
\begin{aligned}
& \operatorname{gr}(\operatorname{Lie} \varphi)\left(\sigma_{1}^{k}\right)\left(Y_{0}\right)=\left[Y_{0}, Y_{k}+Y_{p-k}\right] \\
& \operatorname{gr}(\operatorname{Lie} \varphi)\left(\sigma_{2 i}^{k}\right)\left(Y_{0}\right) \equiv\left[Y_{0},\left[\left[Y_{k}, X\right] X^{2 i-2}\right]-\left[\left[Y_{p-k}, X\right] X^{2 i-2}\right]\right] \bmod I_{3}
\end{aligned}
$$

and

$$
\begin{aligned}
& \operatorname{gr}(\operatorname{Lie} \varphi)\left(\sigma_{2 i+1}^{k}\right)\left(Y_{0}\right) \equiv\left[Y_{0},\left(1-p^{2 i}\right)\left[\left[Y_{k}, X\right] X^{2 i-1}\right]\right. \\
& \left.\quad+\left(1-p^{2 i}\right)\left[\left[Y_{p-k}, X\right] X^{2 i-1}\right]+2 p^{2 i}\left[\left[Y_{0}, X\right] X^{2 i-1}\right]\right] \bmod I_{3}
\end{aligned}
$$

Proof. Assume that $m>1$. After the restriction of $l$-adic polylogarithms to $G_{m}(V, \overrightarrow{01})$ we have the following identities:

$$
p^{m-1}\left(\sum_{k=0}^{p-1} l_{m}\left(\xi_{p}^{k}\right)\right)=l_{m}(\overrightarrow{10}) \quad\left(\xi_{p}^{0}=\overrightarrow{10}\right)
$$

and

$$
l_{m}\left(\xi_{p}^{k}\right)+(-1)^{m} l_{m}\left(\xi_{p}^{p-k}\right)=0 \quad \text { and } \quad l_{2 k}(\overrightarrow{10})=0
$$

(see Part II, Corollaries 11.2.3 and 11.2.6). It follows from the identities $(*)$ and $(* *)$ that

$$
\begin{aligned}
& \text { 5.4.2) } \sum_{k=0}^{p-1} l_{m}\left(\xi_{p}^{p-k}\right)(\sigma)\left[\left[Y_{k}, X\right] X^{m-2}\right] \\
& =\sum_{k=1}^{\frac{p-1}{2}} l_{m}\left(\xi_{p}^{p-k}\right)(\sigma)\left(\left[\left[Y_{k}, X\right] X^{m-2}\right]+(-1)^{m-1}\left[\left[Y_{p-k}, X\right] X^{m-2}\right]\right) \\
& +\frac{p^{m-1}}{1-p^{m-1}}\left(\sum_{k=1}^{\frac{p-1}{2}}\left(l_{m}\left(\xi_{p}^{p-k}\right)(\sigma)+(-1)^{m-1} l_{m}\left(\xi_{p}^{p-k}\right)(\sigma)\right)\right)\left[\left[Y_{0}, X\right] X^{m-2}\right] .
\end{aligned}
$$

It follows from the assumption that the cyclotomic Soulé classes $x^{m}(k)$ in $H^{1}\left(G_{\mathbf{Q}\left(\mu_{p}\right)} ; \mathbf{Q}_{l}(m)\right)$ for $k=1,2, \ldots, \frac{p-1}{2}$ are linearly independent over $\mathbf{Q}_{l}$ and from Corollary 14.3.6 that there are elements $\sigma^{1}, \ldots, \sigma^{\frac{p-1}{2}}$ in $G_{m}(V, \overrightarrow{01})$ such that $l_{m}\left(\xi_{p}^{p-k}\right)\left(\sigma^{j}\right)=0$ if $k \neq j$ and $l_{m}\left(\xi_{p}^{p-k}\right)\left(\sigma^{k}\right) \neq 0$. Hence it follows 
from Lemma 15.3 .2 and the equality (15.4.2) that

$$
\begin{aligned}
& \operatorname{gr}(\operatorname{Lie} \varphi)\left(\sigma^{k}\right)\left(Y_{0}\right) \\
& \equiv\left[Y_{0}, l_{m}\left(\xi_{p}^{p-k}\right)\left(\sigma^{k}\right)\left(\left[\left[Y_{k}, X\right] X^{m-2}\right]+(-1)^{m-1}\left[\left[Y_{p-k}, X\right] X^{m-2}\right]\right)\right. \\
& \left.\quad+\frac{p^{m-1}}{1-p^{m-1}}\left(l_{m}\left(\xi_{p}^{p-k}\right)\left(\sigma^{k}\right)+(-1)^{m-1} l_{m}\left(\xi_{p}^{p-k}\right)\left(\sigma^{k}\right)\right)\left[\left[Y_{0}, X\right] X^{m-2}\right]\right] \\
& \bmod I_{3}+\Gamma^{m+2} \operatorname{Lie}(\mathbf{V}) .
\end{aligned}
$$

Now we set $\sigma_{m}^{k}:=\frac{1}{l_{m}\left(\xi_{p}^{p-k}\right)\left(\sigma^{k}\right)} \sigma^{k}$ in $g r \operatorname{Lie}\left(G_{1}(V, \overrightarrow{01}) / G_{\infty}(V, \overrightarrow{01})\right) \otimes \mathbf{Q}$ if $m$ is even and $\sigma_{m}^{k}:=\frac{1-p^{m-1}}{l_{m}\left(\xi_{p}^{p-k}\right)\left(\sigma^{k}\right)} \sigma^{k}$ in $g r \operatorname{Lie}\left(G_{1}(V, \overrightarrow{01}) / G_{\infty}(V, \overrightarrow{01})\right) \otimes \mathbf{Q}$ if $m$ is odd.

Let us consider the case $m=1$. It follows from the equality $-\xi_{p}^{p-k}$. $\left(1-\xi_{p}^{k}\right)=1-\xi_{p}^{p-k}$ that

$$
l\left(1-\xi_{p}^{k}\right)-l\left(1-\xi_{p}^{p-k}\right)=0
$$

on $G_{1}(V, \overrightarrow{01})$. It follows from [Wa, Theorem 8.9] that the $p$-units $1-\xi_{p}^{k}(k=$ $\left.1, \ldots, \frac{p-1}{2}\right)$ are linearly independent in $\mathbf{Q}\left(\mu_{p}\right)^{*}$. Hence Proposition 14.0.6 implies that the functions $l\left(1-\xi_{p}^{k}\right)\left(k=1, \ldots, \frac{p-1}{2}\right)$ restricted to $G_{\mathbf{Q}\left(\mu_{p \cdot l \infty}\right)}=$ $G_{1}(V, \overrightarrow{01})$ are linearly independent over $\mathbf{Q}_{l}$. Now we finish the proof as for $m>1$.

We shall also use the following notation. If $A$ and $B$ belong to a Lie algebra then we define $\left[A, B^{0}\right]:=A$ and $\left[A, B^{k}\right]:=\left[\left[A, B^{k-1}\right], B\right]$ for $k>0$.

THEOREM 15.4.3. Let $p$ be a prime number greater than 3 and different from $l$. Assume that for each $m>1$ the cyclotomic Soulé classes $x^{m}(k)$ in $H^{1}\left(G_{\mathbf{Q}\left(\mu_{p}\right)} ; \mathbf{Q}_{l}(m)\right)$ for $k=1,2, \ldots, \frac{p-1}{2}$ are linearly independent over $\mathbf{Q}_{l}$. Let $\mathcal{S}$ be a subset of $\{1, \ldots, p-1\}$ satisfying the following conditions

i) if $k \in \mathcal{S}$ then $p-k \in \mathcal{S}$,

ii) $(\mathcal{S}+\mathcal{S}) \cap \mathcal{S}=\emptyset$ (the sum of two elements of $\mathcal{S}$ is calculated mod $p)$.

Then the derivations $\operatorname{gr}(\operatorname{Lie} \varphi)\left(\sigma_{m}^{k_{1}}\right), \ldots, \operatorname{gr}(\operatorname{Lie} \varphi)\left(\sigma_{m}^{k_{q}}\right)$ for $\left\{k_{1}, \ldots, k_{q}\right\} \subset$ $\mathcal{S} \cap\left\{1, \ldots, \frac{p-1}{2}\right\}$ and $m=1,2, \ldots$ generate a free Lie subalgebra of the image of the Lie algebra homomorphism

$$
g r(\operatorname{Lie} \varphi): g r \operatorname{Lie}\left(G_{1}(V, \overrightarrow{01}) / G_{\infty}(V, \overrightarrow{01})\right) \otimes \mathbf{Q} \longrightarrow \operatorname{Der}_{\mathbf{Z} / p}^{*} \operatorname{Lie}(\mathbf{V})
$$

and moreover these derivations are free generators of this Lie subalgebra. 
Proof. We recall that $g r(\operatorname{Lie} \varphi)\left(\sigma_{m}^{k}\right) \in \operatorname{Der}_{\mathbf{Z} / p}^{*} \operatorname{Lie}(\mathbf{V})$ and $g r(\operatorname{Lie} \varphi)$ $\left(\sigma_{m}^{k}\right)\left(Y_{0}\right)=\left[Y_{0}, \beta_{m}^{k}\left(X, Y_{0}, \ldots, Y_{p-1}\right)\right]$ for some $\beta_{m}^{k}=\beta_{m}^{k}\left(X, Y_{0}, \ldots, Y_{p-1}\right) \in$ $\operatorname{Lie}(\mathbf{V})$. To show the theorem it is enough to show that a Lie subalgebra of $(\operatorname{Lie}(\mathbf{V}),\{\})$ generated by $\beta_{1}^{k}, \beta_{2}^{k}, \ldots, \beta_{n}^{k}, \ldots\left(k \in \mathcal{S} \cap\left\{1, \ldots, \frac{p-1}{2}\right\}\right)$ is free and that $\beta_{1}^{k}, \beta_{2}^{k}, \ldots, \beta_{m}^{k}, \ldots\left(k \in \mathcal{S} \cap\left\{1, \ldots, \frac{p-1}{2}\right\}\right)$ are free generators of it.

Let us set

$$
z_{m}^{k}:=\left[Y_{k}, X^{m-1}\right]+(-1)^{m-1}\left[Y_{p-k}, X^{m-1}\right] .
$$

Observe that $\beta_{m}^{k}=z_{m}^{k}+p y_{m}^{k}+\iota(2)_{m}^{k}$, where $y_{m}^{k} \in I_{1}$ and $\iota(2)_{m}^{k} \in I_{2}$. For any arrangement of brackets of length $r$ in $(\operatorname{Lie}(\mathbf{V}),\{\})$ we have

$$
\begin{aligned}
& \left.\left\{\cdots\left\{\beta_{n_{1}}^{k_{1}}, \beta_{n_{2}}^{k_{2}}\right\}, \ldots, \beta_{n_{r}}^{k_{r}}\right\} \cdots\right\} \\
& \left.\quad \equiv\left\{\cdots\left\{z_{n_{1}}^{k_{1}}+p y_{n_{1}}^{k_{1}}, z_{n_{2}}^{k_{2}}+p y_{n_{2}}^{k_{2}}\right\}, \ldots, z_{n_{r}}^{k_{r}}+p y_{n_{r}}^{k_{r}}\right\} \cdots\right\} \bmod I_{r+1} .
\end{aligned}
$$

Let us set $\left.z:=\left\{\cdots\left\{z_{n_{1}}^{k_{1}}+p y_{n_{1}}^{k_{1}}, z_{n_{2}}^{k_{2}}+p y_{n_{2}}^{k_{2}}\right\}, \ldots, z_{n_{r}}^{k_{r}}+p y_{n_{r}}^{k_{r}}\right\} \cdots\right\}$.

We denote by $\operatorname{Lie}(\mathbf{V} ; \mathbf{Z})$ a Lie subalgebra over $\mathbf{Z}$ of $\operatorname{Lie}(\mathbf{V})$ generated by the set $\mathbf{V}$.

The elements $z_{m}^{k}$ and $y_{m}^{k}$ have integer coefficients with respect to a base of Lie( $\mathbf{V})$ given by basic Lie elements in free generators $X, Y_{0}, \ldots, Y_{p-1}$, hence we can view them as elements of $\operatorname{Lie}(\mathbf{V} ; \mathbf{Z})$. Therefore $z$ has also integer coefficients with respect to this base. Observe that

$$
\left.z \equiv\left\{\cdots\left\{z_{n_{1}}^{k_{1}}, z_{n_{2}}^{k_{2}}\right\}, \ldots, z_{n_{r}}^{k_{r}}\right\} \cdots\right\} \bmod p \operatorname{Lie}(\mathbf{V} ; \mathbf{Z}) .
$$

The quotient Lie algebra $\operatorname{Lie}(\mathbf{V} ; \mathbf{Z}) / p \operatorname{Lie}(\mathbf{V} ; \mathbf{Z})$ is a free Lie algebra over $\mathbf{Z} / p$ freely generated by the set $\mathbf{V}$. We consider $I_{r}(\mathcal{S})$ as a Lie ideal of $\operatorname{Lie}(\mathbf{V} ; \mathbf{Z}) / p \operatorname{Lie}(\mathbf{V} ; \mathbf{Z})$.

It follows from Lemma 15.2.10 that in $\operatorname{Lie}(\mathbf{V} ; \mathbf{Z}) / p \operatorname{Lie}(\mathbf{V} ; \mathbf{Z})$ we have

$$
\left.\left.\left\{\cdots\left\{z_{n_{1}}^{k_{1}}, z_{n_{2}}^{k_{2}}\right\}, \ldots, z_{n_{r}}^{k_{r}}\right\} \cdots\right\} \equiv\left[\cdots\left[z_{n_{1}}^{k_{1}}, z_{n_{2}}^{k_{2}}\right], \ldots, z_{n_{r}}^{k_{r}}\right] \cdots\right] \bmod I_{r}(\mathcal{S}) .
$$

The elements $z_{1}^{k}, z_{2}^{k}, \ldots\left(k \in \mathcal{S} \cap\left\{1, \ldots, \frac{p-1}{2}\right\}\right)$ are free generators of a free Lie subalgebra of $\operatorname{Lie}(\mathbf{V} ; \mathbf{Z}) / p \operatorname{Lie}(\mathbf{V} ; \mathbf{Z})$. Hence basic Lie elements in $z_{1}^{k}, z_{2}^{k}, \ldots$ $\left(k \in \mathcal{S} \cap\left\{1, \ldots, \frac{p-1}{2}\right\}\right)$ are linearly independent in $\operatorname{Lie}(\mathbf{V} ; \mathbf{Z}) / p \operatorname{Lie}(\mathbf{V} ; \mathbf{Z})$. Therefore it follows from congruences (15.4.4), (15.4.5) and (15.4.6) that basic Lie elements in elements $\beta_{1}^{k}, \beta_{2}^{k}, \ldots\left(k \in \mathcal{S} \cap\left\{1, \ldots, \frac{p-1}{2}\right\}\right)$ in the Lie algebra $(\operatorname{Lie}(\mathbf{V}),\{\})$ are linearly independent in $\operatorname{Lie}(\mathbf{V})$. Hence the elements $\beta_{1}^{k}, \beta_{2}^{k}, \ldots\left(k \in \mathcal{S} \cap\left\{1, \ldots, \frac{p-1}{2}\right\}\right)$ generate a free Lie subalgebra of $(\operatorname{Lie}(\mathbf{V}),\{\})$ and these elements are free generators of this subalgebra. 
Now we shall consider the case $p=3$.

TheOREm 15.4.7. Let $p=3$ and let $l$ be different from 3. Assume that the cyclotomic Soulé classes $x^{n}(1) \in H^{1}\left(G_{\mathbf{Q}\left(\mu_{3}\right)} ; \mathbf{Q}_{l}(n)\right)$ are different from zero for $n>1$. Then the derivations $\operatorname{gr}(\operatorname{Lie} \varphi)\left(\sigma_{1}^{1}\right), \ldots, \operatorname{gr}(\operatorname{Lie} \varphi)\left(\sigma_{n}^{1}\right), \ldots$ generate a free Lie subalgebra of $\operatorname{Im}(\operatorname{gr}(\operatorname{Lie} \varphi))$ and these derivations are free generators of this Lie subalgebra.

Proof. We must modify the proof of Theorem 15.4 .3 for $p=3$. Let $I\left(Y_{0}, Y_{2}\right)$ be a Lie ideal of $\operatorname{Lie}(\mathbf{V} ; \mathbf{Z}) / 3 \operatorname{Lie}(\mathbf{V} ; \mathbf{Z})$ generated by $Y_{0}$ and $Y_{2}$. Then we have

$$
\left.\left.\left\{\cdots\left\{z_{n_{1}}^{1}, z_{n_{2}}^{1}\right\}, \ldots, z_{n_{r}}^{1}\right\} \cdots\right\} \equiv\left[\cdots\left[z_{n_{1}}^{1}, z_{n_{2}}^{1}\right], \ldots, z_{n_{r}}^{1}\right] \cdots\right] \bmod I\left(Y_{0}, Y_{2}\right)
$$

and

$$
\left.\left.\left[\cdots\left[z_{n_{1}}^{1}, z_{n_{2}}^{1}\right], \ldots, z_{n_{r}}^{1}\right] \cdots\right] \equiv\left[\cdots\left[s_{n_{1}}^{1}, s_{n_{2}}^{1}\right], \ldots, s_{n_{r}}^{1}\right] \cdots\right] \bmod I\left(Y_{0}, Y_{2}\right),
$$

where $s_{m}^{1}=\left[Y_{1}, X^{m-1}\right]$. The congruences (15.4.8) and (15.4.9) replace the congruence (15.4.6). The rest of the proof is the same as the proof of Theorem 15.4.3.

Conjecture 15.4.10. Let $p$ be a prime number greater than 2 and different from $l$. The derivations $\operatorname{gr}(\operatorname{Lie} \varphi)\left(\sigma_{1}^{k}\right), \operatorname{gr}(\operatorname{Lie} \varphi)\left(\sigma_{2}^{k}\right), \operatorname{gr}(\operatorname{Lie} \varphi)\left(\sigma_{3}^{k}\right)$, $\ldots, g r(\operatorname{Lie} \varphi)\left(\sigma_{n}^{k}\right), \ldots$ for $k=1, \ldots, \frac{p-1}{2}$ generate $\operatorname{Im}(\operatorname{gr}(\operatorname{Lie} \varphi))$.

15.5. Now we shall consider the case when $p=2$. In fact the proof will repeat the arguments from [D2] with necessary modifications for the $l$-adic case. Let us set $V:=\mathbf{P}_{\mathbf{Q}}^{1} \backslash\{0,1,-1, \infty\}$. Let $\overrightarrow{01}$ be a base point of the fundamental group. We choose a family $\Gamma=\left\{\gamma_{0}, \gamma_{1}, \gamma_{\infty}\right\}$ as on Picture 4.

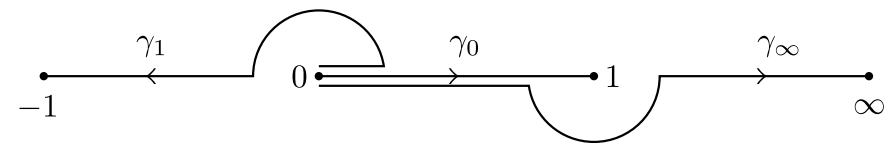

Picture 4

Let $\left(x, y_{0}, y_{1}, z\right)$ be a sequence of geometric generators of $\pi_{1}(V(\mathbf{C}) ; \overrightarrow{01})$ corresponding to the family $\Gamma$. 
Lemma 15.5.1. Let $\sigma \in G_{m}(V, \overrightarrow{01})$. If $m>1$ then

$$
\begin{aligned}
& \log \left(\Lambda_{\gamma_{0}}(\sigma)\left(X, Y_{0}, Y_{1}\right)\right)=l_{m}(\overrightarrow{10})(\sigma)\left[\left[Y_{0}, X\right] X^{m-2}\right] \\
& \quad+\left(\frac{1}{2^{m-1}}-1\right) l_{m}(\overrightarrow{10})(\sigma)\left[\left[Y_{1}, X\right] X^{m-2}\right] \bmod I_{2}+\Gamma^{m+1} L(\mathbf{V})
\end{aligned}
$$

If $m=1$ then

$$
\log \left(\Lambda_{\gamma_{0}}(\sigma)\left(X, Y_{0}, Y_{1}\right)\right)=l(2)(\sigma) Y_{1} \bmod \Gamma^{2} L(\mathbf{V})
$$

Proof. The lemma follows from Lemma 15.3.1 and the identity

$$
2^{m-1}\left(l_{m}(\overrightarrow{10})+l_{m}(-1)\right)=l_{m}(\overrightarrow{10})
$$

(see Part II, Corollary 11.2.3).

LEMMA 15.5.2. In the Lie algebra $\operatorname{gr} \operatorname{Lie}\left(G_{1}(V, \overrightarrow{01}) / G_{\infty}(V, \overrightarrow{01})\right) \otimes \mathbf{Q}$ there are elements $\sigma_{1}, \sigma_{3}, \sigma_{5}, \ldots, \sigma_{2 m+1}, \ldots$ such that any $\sigma_{k}$ is homogenous of degree $k, g r(\operatorname{Lie} \varphi)\left(\sigma_{1}\right)\left(Y_{0}\right)=\left[Y_{0}, Y_{1}\right]$ and

$$
\begin{aligned}
& \operatorname{gr}(\operatorname{Lie} \varphi)\left(\sigma_{2 m+1}\right)\left(Y_{0}\right) \\
& \quad=\left[Y_{0}, 2^{2 m}\left[\left[Y_{0}, X\right] X^{2 m-1}\right]+\left(1-2^{2 m}\right)\left[\left[Y_{1}, X\right] X^{2 m-1}\right]\right] \bmod I_{3} .
\end{aligned}
$$

Proof. The homomorphism $l_{n}(\overrightarrow{10})$ for $n$ odd and greater than 1 is a generator of $\operatorname{Hom}_{\mathbf{Z}_{l}^{*}}\left(G_{\mathbf{Q}\left(\mu_{l} \infty\right)} ; \mathbf{Z}_{l}(n)\right) \simeq \mathbf{Z}_{l}$ (see $\left.[\mathrm{IS}]\right)$. Hence it follows from Part I, Lemma 3.0 .8 that $l_{n}(\overrightarrow{10})$ restricted to $G_{n}(V, \overrightarrow{01})$ is different from zero. Therefore there is $\sigma(n) \in G_{n}(V, \overrightarrow{01})$ such that $l_{n}(\overrightarrow{10})(\sigma(n)) \neq$ 0 for $n$ odd and greater than 1. We set $\sigma_{n}=\frac{2^{n-1}}{l_{n}(\overrightarrow{10})(\sigma(n))} \cdot \sigma(n)$ in $\operatorname{gr} \operatorname{Lie}\left(G_{1}(V, \overrightarrow{01}) / G_{\infty}(V, \overrightarrow{01})\right) \otimes \mathbf{Q}$ if $n$ is odd and greater than 1 .

The homomorphism $l(2)$ is a Kummer character associated to 2. Hence there is $\sigma(1) \in G_{1}(V, \overrightarrow{01})$ such that $l(2)(\sigma(1)) \neq 0$. We set $\sigma_{1}=\frac{1}{l(2)(\sigma(1))}$. $\sigma(1)$ in $g r \operatorname{Lie}\left(G_{1}(V, \overrightarrow{01}) / G_{\infty}(V, \overrightarrow{01})\right) \otimes \mathbf{Q}$.

TheOREM 15.5.3. The derivations $\operatorname{gr}(\operatorname{Lie} \varphi)\left(\sigma_{1}\right), \operatorname{gr}(\operatorname{Lie} \varphi)\left(\sigma_{3}\right), \ldots$, $\operatorname{gr}(\operatorname{Lie} \varphi)\left(\sigma_{2 m+1}\right), \ldots$ generate a free Lie subalgebra of $\operatorname{Im}(\operatorname{gr}(\operatorname{Lie} \varphi))$ and these derivations are free generators of this Lie subalgebra.

Proof. We have $\operatorname{gr}(\operatorname{Lie} \varphi)\left(\sigma_{1}\right)\left(Y_{0}\right)=\left[Y_{0}, Y_{1}\right]$ and $\operatorname{gr}(\operatorname{Lie} \varphi)\left(\sigma_{2 n+1}\right)\left(Y_{0}\right)$ $=\left[Y_{0}, \beta_{2 n+1}\right]$ for $n>0$, where $\beta_{2 n+1} \in \operatorname{Lie}(\mathbf{V})$ is homogenous of degree 
$2 n+1$. The Lie algebra $\operatorname{Der}_{Z / 2}^{*} \operatorname{Lie}(\mathbf{V})$ is isomorphic to $\left(\operatorname{Lie}(\mathbf{V}) /\left\langle Y_{0}\right\rangle,\{\}\right)$. Hence it is enough to show that a Lie subalgebra of $(\operatorname{Lie}(\mathbf{V}),\{\})$ generated by $\beta_{1}, \beta_{3}, \ldots, \beta_{2 n+1}, \ldots$ is free and it is freely generated by these elements. Observe that $\beta_{2 n+1}=z_{2 n+1}+y_{2 n+1}+\iota_{2 n+1}$ where $z_{2 n+1}=\left[Y_{1}, X^{2 n}\right]\left(z_{1}=\right.$ $\left.Y_{1}\right), y_{2 n+1}=2^{2 n}\left[Y_{0}, X^{2 n}\right]-2^{2 n}\left[Y_{1}, X^{2 n}\right]\left(y_{1}=0\right)$ and $\iota_{2 n+1} \in I_{2}$.

For any arrangement of brackets of length $r$ in $(\operatorname{Lie}(\mathbf{V}),\{\})$ we have

$$
\begin{aligned}
& \left.\left\{\cdots\left\{\beta_{n_{1}}, \beta_{n_{2}}\right\}, \ldots, \beta_{n_{r}}\right\} \cdots\right\} \\
& \left.\quad \equiv\left\{\cdots\left\{z_{n_{1}}+y_{n_{1}}, z_{n_{2}}+y_{n_{2}}\right\}, \ldots, z_{n_{r}}+y_{n_{r}}\right\} \cdots\right\} \bmod I_{r+1} .
\end{aligned}
$$

Let us set $\left.z:=\left\{\cdots\left\{z_{n_{1}}+y_{n_{1}}, z_{n_{2}}+y_{n_{2}}\right\}, \ldots, z_{n_{r}}+y_{n_{r}}\right\} \cdots\right\}$. The elements $z_{m}$ and $y_{m}$ have integer coefficients with respect to a base of $\operatorname{Lie}(\mathbf{V})$ given by basic Lie elements in free generators $X, Y_{0}$ and $Y_{1}$. Hence $z$ has integer coefficients with respect to this base. We consider these elements as elements of $\operatorname{Lie}(\mathbf{V} ; \mathbf{Z})$, where $\operatorname{Lie}(\mathbf{V} ; \mathbf{Z})$ is a Lie subalgebra over $\mathbf{Z}$ of $\operatorname{Lie}(\mathbf{V})$ generated by $X, Y_{0}$ and $Y_{1}$. Observe that

$$
\left.z \equiv\left\{\cdots\left\{z_{n_{1}}, z_{n_{2}}\right\}, \ldots, z_{n_{r}}\right\} \cdots\right\} \bmod 2 \operatorname{Lie}(\mathbf{V} ; \mathbf{Z}) .
$$

The quotient Lie algebra $\operatorname{Lie}(\mathbf{V} ; \mathbf{Z}) / 2 \operatorname{Lie}(\mathbf{V} ; \mathbf{Z})$ is a free Lie algebra over $Z / 2$ on free generators $X, Y_{0}$ and $Y_{1}$. Let $\mathcal{S}=\{1\}$. We recall that $I_{r}(\mathcal{S})$ is a Lie ideal of $\operatorname{Lie}(\mathbf{V} ; \mathbf{Z}) / 2 \operatorname{Lie}(\mathbf{V} ; \mathbf{Z})$ generated by Lie brackets which contain at least $r Y^{\prime}$ 's and at least one of them is $Y_{1}$ and at least one of them is $Y_{0}$.

Observe that

$$
\left.\left.\left\{\cdots\left\{z_{n_{1}}, z_{n_{2}}\right\}, \ldots, z_{n_{r}}\right\} \cdots\right\} \equiv\left[\cdots\left[z_{n_{1}}, z_{n_{2}}\right], \ldots, z_{n_{r}}\right] \cdots\right] \bmod I_{r}(\mathcal{S}) .
$$

The elements $z_{1}, z_{3}, \ldots, z_{2 n+1}, \ldots$ are free generators of a free Lie subalgebra of $\operatorname{Lie}(\mathbf{V} ; \mathbf{Z}) / 2 \operatorname{Lie}(\mathbf{V} ; \mathbf{Z})$. Hence basic Lie elements of degree $r$ in $z_{1}, z_{3}, \ldots, z_{2 n+1}, \ldots$ are linearly independent in $\operatorname{Lie}(\mathbf{V} ; \mathbf{Z}) / 2 \operatorname{Lie}(\mathbf{V} ; \mathbf{Z})$. Therefore it follows from congruences (15.5.4), (15.5.5) and (15.5.6) that basic Lie elements of degree $r$ in elements $\beta_{1}, \beta_{3}, \ldots, \beta_{2 n+1}, \ldots$ in the Lie algebra $(\operatorname{Lie}(\mathbf{V}),\{\})$ are linearly independent in $\operatorname{Lie}(\mathbf{V})$. Hence the elements $\beta_{1}, \beta_{3}, \ldots, \beta_{2 n+1}, \ldots$ generate a free Lie subalgebra of $(L(\mathbf{V}),\{\})$ and these elements are free generators of this Lie subalgebra. (This proof is essentially the repetition of the proof given by Deligne for the Hodge realization (see [D2]).)

Conjecture 15.5.7. The derivations $l\left(\sigma_{1}\right), l\left(\sigma_{3}\right), \ldots, l\left(\sigma_{2 n+1}\right), \ldots$ generate $\operatorname{Im}(\operatorname{gr}(\operatorname{Lie} \varphi))$. 
15.6. Now we shall assume that $n$ is a power of a prime number. Let $p$ be a prime number different from $l$ and let $n=p^{r}$. Let $V=\mathbf{P}_{\mathbf{Q}\left(\mu_{n}\right)}^{1} \backslash$ $\left\{0, \mu_{n}, \infty\right\}$.

Proposition 15.6.1. Assume that for each $i>1$ the cyclotomic Soule classes $x^{i}(k) \in H^{1}\left(G_{\mathbf{Q}\left(\mu_{n}\right)} ; \mathbf{Q}_{l}(i)\right)$ for $0<k<\frac{n}{2}$ and $(k, p)=1$ are linearly independent over $\mathbf{Q}_{l}$. Then in the Lie algebra $\operatorname{gr} \operatorname{Lie}\left(G_{1}(V, \overrightarrow{01}) / G_{\infty}(V, \overrightarrow{01})\right) \otimes$ $\mathbf{Q}$ there are elements $\sigma_{m+1}^{k}$ for $m=1,2, \ldots$ and for $0<k<\frac{n}{2}$ and $(k, p)=$ 1 such that $\sigma_{m+1}^{k}$ is homogenous of degree $m+1$ and $g r(\operatorname{Lie} \varphi)\left(\sigma_{m+1}^{k}\right)\left(Y_{0}\right)=$ $\left[Y_{0}, \beta_{m+1}^{k}\right]$, where

$$
\begin{aligned}
\beta_{m+1}^{k}= & \beta_{m+1}^{k}\left(X, Y_{0}, \ldots, Y_{n-1}\right) \\
= & \left(1-p^{r m}\right)\left(\left[Y_{k}, X^{m}\right]+(-1)^{m}\left[Y_{n-k}, X^{m}\right]\right) \\
& +p^{m} \sum_{j=1(j, p) \geq p}^{n-1} d_{m+1, k}^{j}\left[Y_{j}, X^{m}\right]+p^{r m} d_{m+1, k}^{0}\left[Y_{0}, X^{m}\right] \bmod I_{2}
\end{aligned}
$$

and where $d_{m+1, k}^{j}$ are integers.

Proposition 15.6.2. Assume that for each $i>1$ the cyclotomic Soulé classes $x^{i}(k) \in H^{1}\left(G_{\mathbf{Q}\left(\mu_{n}\right)} ; \mathbf{Q}_{l}(i)\right)$ for $0<k<\frac{n}{2}$ and $(k, p)=1$ are linearly independent over $\mathbf{Q}_{l}$. Let $\mathcal{S}$ be a subset of $\{i \mid 0<i<n$ and $(i, p)=1\}$ satisfying the following conditions

i) if $k \in \mathcal{S}$ then $n-k \in \mathcal{S}$,

ii) $(\mathcal{S}+\mathcal{S}) \cap \mathcal{S}=\emptyset$ (the sum of two elements of $\mathcal{S}$ is calculated mod $n)$.

Then the derivations $\operatorname{gr}(\operatorname{Lie} \varphi)\left(\sigma_{m+1}^{k_{1}}\right), \ldots, \operatorname{gr}(\operatorname{Lie} \varphi)\left(\sigma_{m+1}^{k_{q}}\right)$ for $\left\{k_{1}, \ldots, k_{q}\right\}$ $\subset \mathcal{S} \cap\left[1, \frac{n}{2}\right]$ and for $m=1,2, \ldots$ generate a free Lie subalgebra of $\operatorname{Im}(\operatorname{gr}(\operatorname{Lie} \varphi))$ and these derivations are free generators of this Lie subalgebra.

Corollary 15.6.3. Let $n=2^{r}$. Assume that for each $i>1$ the cyclotomic Soulé classes $x^{i}(k) \in H^{1}\left(G_{\mathbf{Q}\left(\mu_{n}\right)} ; \mathbf{Q}_{l}(i)\right)$ for $0<k<\frac{n}{2}$ and $k$ odd are linearly independent over $\mathbf{Q}_{l}$. Then the derivations $g r(\operatorname{Lie} \varphi)\left(\sigma_{m+1}^{k}\right)$ for $m=1,2, \ldots$ and for $0<k<\frac{n}{2}$ and $k$ odd generate a free Lie subalgebra of $\operatorname{Im}(\operatorname{gr}(\operatorname{Lie} \varphi))$ and these derivations are free generators of this Lie subalgebra. 
The proofs of 15.6.1-15.6.3 are the same as proofs of 15.4.1 and 15.4.3. We do not include in our considerations derivations in degree 1 because working modulo $p$ we are not able to eliminate $Y_{j}$ for $(j, p) \geq p$.

Corollary 15.6.4. Let $n=4$. Assume that for each $m>0$ the cyclotomic Soulé class $x^{2 m}(1) \in H^{1}\left(G_{\mathbf{Q}(i)} ; \mathbf{Q}_{l}(2 m)\right)$ does not vanish. Then the derivations $\operatorname{gr}(\operatorname{Lie} \varphi)\left(\sigma_{m}^{1}\right)(m=1,2,3, \ldots)$ are free generators of a free Lie subalgebra of $\operatorname{Im}(\operatorname{gr}(\operatorname{Lie} \varphi))$.

Proof. We have $(1-i)(1+i)=2$ and $i(1-i)=1+i$. This implies that $l(2)=2 l(1-i)$. Hence we can include also the derivations in degree 1 in our considerations.

Proposition 15.6.5. Let $n=8$. Assume that for any $m>1$ the $l$ adic polylogarithms $l_{m}\left(\xi_{8}\right)$ and $l_{m}\left(\xi_{8}^{3}\right)$ are linearly independent. Then the derivations $\operatorname{gr}(\operatorname{Lie} \varphi)\left(\sigma_{m}^{1}\right), \operatorname{gr}(\operatorname{Lie} \varphi)\left(\sigma_{m}^{3}\right)$ for $m=1,2,3, \ldots$ are free generators of a free Lie subalgebra of $\operatorname{Im}(\operatorname{gr}(\operatorname{Lie} \varphi))$.

Proof. It follows from Corollary 15.6.3 that the most interesting derivations are in degree 1 . Let $\sigma \in G_{1}(V, \overrightarrow{01})$. Lemma 15.3.1 implies that

$$
\log \left(\Lambda_{\gamma_{0}}(\sigma)\left(X, Y_{0}, Y_{1}, \ldots, Y_{7}\right)\right) \equiv \sum_{k=1}^{7} l\left(1-\xi_{8}^{8-k}\right)(\sigma) Y_{k} \bmod \Gamma^{2} L(\mathbf{V})
$$

Observe that $\left(1-\xi_{8}^{2}\right)=\left(1-\xi_{8}^{1}\right) \cdot\left(1-\xi_{8}^{5}\right),\left(1-\xi_{8}^{6}\right)=\left(1-\xi_{8}^{3}\right) \cdot\left(1-\xi_{8}^{7}\right)$, $\left(1-\xi_{8}^{4}\right)=\left(1-\xi_{8}^{1}\right) \cdot\left(1-\xi_{8}^{3}\right) \cdot\left(1-\xi_{8}^{5}\right) \cdot\left(1-\xi_{8}^{7}\right)$ and $-\xi_{8}^{8-k} \cdot\left(1-\xi_{8}^{k}\right)=\left(1-\xi_{8}^{8-k}\right)$. The 2 -units $\left(1-\xi_{8}^{1}\right)$ and $\left(1-\xi_{8}^{3}\right)$ are linearly independent in $\mathbf{Q}\left(\mu_{8}\right)^{*}$. Hence in degree 1 we have two linearly independent derivations $g r(\operatorname{Lie} \varphi)\left(\sigma_{1}^{1}\right)$ and $\operatorname{gr}(\operatorname{Lie} \varphi)\left(\sigma_{1}^{3}\right)$ such that

$$
\operatorname{gr}(\operatorname{Lie} \varphi)\left(\sigma_{1}^{1}\right)\left(Y_{0}\right)=\left[Y_{0}, Y_{1}+Y_{7}+Y_{2}+Y_{6}+2 Y_{4}\right]
$$

and

$$
g r(\operatorname{Lie} \varphi)\left(\sigma_{1}^{3}\right)\left(Y_{0}\right)=\left[Y_{0}, Y_{3}+Y_{5}+Y_{2}+Y_{6}+2 Y_{4}\right]
$$

After passing to Lie algebra $(\operatorname{Lie}(\mathbf{V} ; \mathbf{Z}) / 2 \operatorname{Lie}(\mathbf{V} ; \mathbf{Z}),\{\})$ we need to study a Lie subalgebra generated by elements $z_{1}^{1}:=Y_{1}+Y_{7}+Y_{2}+Y_{6}, z_{1}^{3}:=Y_{3}+$ $Y_{5}+Y_{2}+Y_{6}$ and $z_{m}^{1}:=\left[Y_{1}, X^{m-1}\right]+(-1)^{m-1}\left[Y_{7}, X^{m-1}\right], z_{m}^{3}:=\left[Y_{3}, X^{m-1}\right]+$ $(-1)^{m-1}\left[Y_{5}, X^{m-1}\right]$ for $m=2,3,4, \ldots$. 
Let $\operatorname{Lie}\left(X, Y_{1}, Y_{5} ; \mathbf{Z} / 2\right)$ be a Lie subalgebra of $\operatorname{Lie}(\mathbf{V} ; \mathbf{Z}) / 2 \operatorname{Lie}(\mathbf{V} ; \mathbf{Z})$ generated by $X, Y_{1}$ and $Y_{5}$. Let $J$ be a Lie ideal of $\operatorname{Lie}(\mathbf{V} ; \mathbf{Z}) / 2 \operatorname{Lie}(\mathbf{V} ; \mathbf{Z})$ generated by $Y_{0}, Y_{2}, Y_{3}, Y_{4}, Y_{6}$ and $Y_{7}$. Observe that

$$
\operatorname{Lie}(\mathbf{V} ; \mathbf{Z}) / 2 \operatorname{Lie}(\mathbf{V} ; \mathbf{Z}) \simeq \operatorname{Lie}\left(X, Y_{1}, Y_{5} ; \mathbf{Z} / 2\right) \oplus J .
$$

Let us set

$$
s_{m}^{1}:=\left[Y_{1}, X^{m-1}\right] \quad \text { and } s_{m}^{3}:=\left[Y_{5}, X^{m-1}\right]
$$

for $m=1,2,3, \ldots$ One shows that for any Lie bracket of elements $z_{m}^{1}, z_{m}^{3}$ for $m=1,2,3, \ldots$ of length $r$ we have

$$
\left\{\cdots\left\{z_{m_{1}}^{i_{1}}, z_{m_{2}}^{i_{2}}\right\}, \ldots, z_{m_{r}}^{i_{r}}\right\} \equiv\left[\cdots\left[s_{m_{1}}^{i_{1}}, s_{m_{2}}^{i_{2}}\right], \ldots, s_{m_{r}}^{i_{r}}\right] \bmod J
$$

The elements $s_{m}^{1}$ and $s_{m}^{3}$ for $m=1,2,3, \ldots$ are free generators of a free Lie subalgebra of $\operatorname{Lie}\left(X, Y_{1}, Y_{5} ; \mathbf{Z} / 2\right)$. This implies that the elements $z_{m}^{1}$, $z_{m}^{3}$ for $m=1,2,3, \ldots$ are free generators of a free Lie subalgebra of $(\operatorname{Lie}(\mathbf{V} ; \mathbf{Z}) / 2 \operatorname{Lie}(\mathbf{V} ; \mathbf{Z}),\{\})$. This implies the proposition.

Remark 15.6.6. According to [G, p. 427], the results for $n=3$ and $n=4$ are also given in Deligne's letter to Goncharov.

\section{REFERENCES}

[D1] P. Deligne, Le groupe fondamental de la droite projective moins trois points, Galois Groups over Q (Y. Ihara, K. Ribet and J.-P. Serre, eds.), Mathematical Sciences Research Institute Publications, no 16 (1989), pp. 79-297.

[D2] P. Deligne, talk on the conference in Schloss Ringberg (1998).

[G] A. Goncharov, The dihedral Lie algebra and Galois symmetries of $\pi_{1}^{(l)}\left(\mathbf{P}^{1} \backslash(\{0, \infty\}\right.$ $\left.\cup \mu_{N}\right)$ ), Duke Math. J., 110 (2001), 397-486.

[HW] A. Huber and J. Wildeshaus, Classical motivic polylogarithm according to Beilinson and Deligne, Documenta Mathematica, 3 (1998), 27-113.

[I1] Y. Ihara, Profinite braid groups, Galois representations and complex multiplications, Annals of Math., 123 (1986), 43-106.

[I2] Y. Ihara, Braids, Galois Groups and Some Arithmetic Functions, Proc. of the Int. Cong. of Math. Kyoto (1990), 99-119.

[IS] H. Ichimura and K. Sakaguchi, The Non-Vanishing of a Certain Kummer Character $\chi_{m}$ (after Soulé) and Some Related Topics, Advanced Studies in Pure Mathematics 12 (1987), pp. 53-64.

[NW] H. Nakamura and Z. Wojtkowiak, On explicit formulae for l-adic polylogarithms, Arithmetic Fundamental Groups and Noncommutative Algebra (M. Fried and Y. Ihara, eds.), Proceedings of Symposia in Pure Mathematics, Vol. 70, American Mathematical Society, pp. 285-294. 
[S1] Ch. Soulé, K-théorie des anneaux d'entiers de corps de nombres et cohomologie etale, Inventiones math., 55 (1979), 251-295.

[S2] Ch. Soulé, On higher p-adic regulators, Springer Lecture Notes N 854 (1981), 372-401.

[S3] Ch. Soulé, Eléments Cyclotomiques en K-Théorie, Asterisque, 147-148 (1987), $225-258$.

[Wa] L. C. Washington, Introduction to Cyclotomic Fields, Springer-Verlag, 1997.

[W1] Z. Wojtkowiak, Monodromy of iterated integrals and Galois action on the fundamental group of a projective line minus a finite number of points, Prépublication n 578, Université de Nice, 2000.

[W2] Z. Wojtkowiak, A note on functional equations of l-adic polylogarithms, Journal of the Inst. of Math. Jussieu, 3 (2004), no. 3, 461-471.

[W3] Z. Wojtkowiak, Non-Abelian Unipotent Periods and Monodromy of Iterated Integrals, Journal of the Inst. of Math. Jussieu, 2 (2003), no. 1, 1-24.

Université de Nice-Sophia Antipolis

Département de Mathématiques

Laboratoire Jean Alexandre Dieudonné

U.R.A. au C.N.R.S., No 168

Parc Valrose - B.P.N $N^{\circ} 71$

06108 Nice Cedex 2

France

wojtkow@math.unice.fr 